\title{
Quantum Model of Gravity Unifies Relativistic Effects, Describes Inflation/Expansion Transition, Matches CMB Data
}

\author{
Jody A. Geiger \\ Informativity Institute, Chicago, Illinois, USA \\ Email: jodygeiger@informativity.org
}

How to cite this paper: Geiger, J.A. (2018) Quantum Model of Gravity Unifies Relativistic Effects, Describes Inflation/Expansion Transition, Matches CMB Data. Journal of High Energy Physics, Gravitation and Cosmology, 4, 655-694.

https://doi.org/10.4236/jhepgc.2018.44038

Received: July 17, 2018

Accepted: October 8, 2018

Published: October 11, 2018

Copyright $\odot 2018$ by author and Scientific Research Publishing Inc. This work is licensed under the Creative Commons Attribution International License (CC BY 4.0).

http://creativecommons.org/licenses/by/4.0/

\begin{abstract}
Presenting a unified model of motion and gravity has proved difficult as current approaches to quantum and classical physics are incompatible. Using measurement quantization-a model that demonstrates the physical significance of Planck's units of length, mass, and time-measure is expressed as counts of the fundamental units establishing a common framework for describing quantum and cosmological phenomena with expressions that are defined throughout the entire physical domain. Beginning with the Pythagorean Theorem, we demonstrate an understanding of measure with respect to static and moving references. The model is extended to include the measure of mass thus completing a single approach for describing the contraction and dilation of measure. With this new approach, relativistic effects are now described as properties of quantized finite units of measure. In support of the model, several descriptions of phenomena are resolved that match our most precise data such as the measure of dark energy, universal expansion, mass distribution, and the age of the Cosmic Microwave Background.
\end{abstract}

\section{Keywords}

Relativity, Measurement Distortion, Length Contraction, Time Dilation, Quantum Gravity, Gravitational Constant, Planck Units, Dark Energy, Dark Matter, Cosmic Microwave Background, Space-Time Curvature

\section{Introduction}

We shall use the principles of Informativity [1], an approach that recognizes the countable nature of discrete units of measure, to describe the contraction and dilation of measure with respect to objects in motion and in gravitational fields. 
The approach is based on the quantization of measure and has been successful in several disciplines with more than 20 physical predictions. Where not constrained by the precision of measurement data, a six-sigma correspondence is demonstrated (i.e. $\theta_{s i}=3.26239$ rad. corresponds to the $6^{\text {th }}$ digit, ([1], Equation (19))). Valid throughout the entire measurement domain, the quantization of measure has allowed the development of a quantum model of gravity.

Extending this work, we now present new expressions for the contraction and dilation of measure with respect to motion and gravity. We intentionally distinguish the approach from relativity as this approach does not take the same path presented by Einstein. Specifically, the approach is quantum, a description of phenomena as counts of physically significant units of measure.

The approach uses only the Pythagorean Theorem and the aforementioned quantum description of gravity. Equivalence is an outcome, a prediction of the model in support of the axioms on which General Relativity (GR) [2] is premised. Notably, the approach succeeds in one area where GR does not. All expressions are valid for the entire measurement domain. There are no examples of division by zero or infinity.

We shall also use the quantization of measure to describe properties of several other phenomena, for instance, why space demonstrates curvature. Calculations affected by the quantization of measure, such as the age of the Cosmic Microwave Background ( $\mathrm{CMB})$, the mass/energy associated with dark energy, and the respective mass distributions for visible, observable, and non-observable mass are each shown to match our best measurement data. While not directly related to the contraction and dilation of measure, we also describe the transition event that causes inflation to end and expansion of the universe to begin.

Lastly, while this paper may be understood with the information presented within, many of the expressions used are new to modern physics, first published in the Journal of High Energy Physics, Gravitation and Cosmology under the title "Measurement Quantization Unites Classical and Quantum Physics" [1]. This paper is an extension of the discoveries and supporting evidence presented in the prior. Readers will find a firmer footing, by starting there.

\subsection{Theoretical Landscape}

Informativity differs dramatically from the theoretical landscape in one important way.

Informativity is a model built on the idea that measure is discrete and that nature is non-discrete.

The discreteness of measure is a physically significant property of observation ([1], see Equations (20)-(22), (31), (47), (57)). Over the last 100 years, several models have gained favor, namely the Standard Model, Quantum Mechanics, Special Relativity (SR), General Relativity (GR), Supersymmetry (SUSY), String Theory, Loop Quantum Gravity (LQG), Modified Newtonian Dynamics (MOND), $\Lambda \mathrm{CDM}$ and several lesser known models. Each demonstrates success from a different point of view, but, in specific situations, they 
break down. In an effort to unravel the puzzle a process of scope restriction, "fitting" and sometimes perturbation theory are applied to align or approximate expressions with what is observed. We ask how can these models be accurate throughout most of the physical domain, yet inaccurate elsewhere?

\subsection{Axioms}

Axioms set the foundation of a model. In turn, expressions are defined and interpreted with respect to those axioms. By example, most models follow one of these two axioms.

- Nature is discrete.

- Nature is non-discrete.

To better understand the effects of axiom selection we may consider the expression $v=\Delta x / \Delta t$. The expression is true for all macroscopic measure regardless of the selected axiom, but what about a description in the quantum domain ... a velocity equal to $10^{-1000} \mathrm{~m} / \mathrm{s}$. In this case, the value will be physically significant in a non-discrete universe. But, if nature is discrete, that is quantized, then at what velocity is the quantization physically significant? Length and time will have invariant minimums. And as such, the measure may not be physically significant.

The property of discreteness is relevant to all theories. Specifically, the Standard Model, GR/SR, $\Lambda$ CDM, SUSY, String Theory and MOND are each built on the idea that nature is non-discrete. Quantum Mechanics and LQG, in contrast, are built on the idea that nature is discrete or at least some part of it. Informativity ascribes discreteness to a new consideration-measure-departing from the existing theoretical landscape.

The development was a necessity that arose from a simple gedanken as described in Figure 1. That led to expressions that describe gravity as the continuous loss of a remainder count above and beyond a whole unit count of a more precisely calculated distance count of a reference where $c=\left(1^{2}+b^{2}\right)^{1 / 2}$. The idea is that measure is reference dependent and as such any remainder count $Q_{L}$ less than the reference $l_{f}$ cannot be measured and is lost at each fundamental unit of time $t_{f}$ The axiom, therefore, is that measure is discrete and that nature is non-discrete. A distinction between what we measure and what is nature is new to our understanding of the behavior of matter. In the case of gravity, these

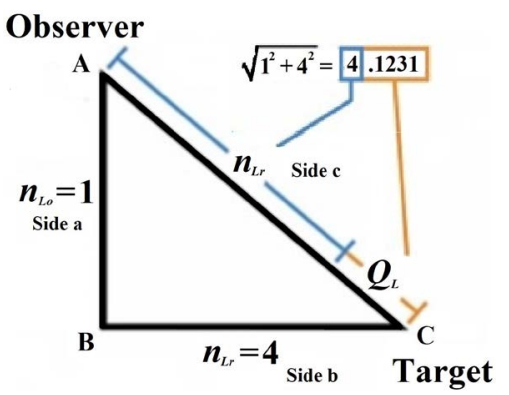

Figure 1. Counts of $l_{f}$ between a target and a center of gravity. 
expressions describe what is measured and what is measured describes what is observed.

The existence of physically significant fundamental measures, $l_{f}, m_{f}$ and $t_{f}$ carries with it an implied physical scope where measure is valid. Measurement scope in turn describes how information is constrained, and thus what the observer experiences, for instance, neatly dividing the matter we observe into that which is visible, observable, dark and never to be observed (i.e. because the light from that matter will never reach a given point in space-time) ([1], Equations (109), (110), (113), (115)). The frequency bounds between the measures set both upper and lower bounds to observation, not just between length and time, $c=$ $l_{f} / t_{f}$ but between mass and time, $m_{f} / t_{f}$ and between mass and length, $m_{f} / l_{f}$ Consider the expression for escape velocity,

$$
v_{e}=\left(2 \frac{G M}{r}\right)^{1 / 2}
$$

Reduced to a fundamental nomenclature where $c=l_{f} f t_{f}, r=n_{L r} l_{f}, M=n_{M} m_{f}$ and $G=\left(l_{f} / t_{f}\right)^{3}\left(t_{f} / m_{f}\right)([1]$, Equation (31)), we consider the case at the surface of a black hole, $V_{e}=\mathrm{c}$,

$$
n_{L r}=2 n_{M}
$$

Without a bound to the amount of mass $M$ in a given radius $r$, the Standard Model is unbounded in scope. LQG, conversely, is protected by its reliance on a discrete space-time. But, LQG does not establish physical evidence [3] for the discreteness of nature nor does it utilize expression operators that preserve the discrete elements being described.

Consider now the change in position of a particle in a gravitational field. Notably, the change is less than the Planck length $I_{f}$ during any given unit of Planck time $t_{f}$ Since we know $l_{f}$ and $t_{f}$ to be bounded, that is that each may not take on a value where $l_{f} / t_{f}$ is greater than $c$, we must concede that space-time (nature) defies the idea of discreteness. Nevertheless, there are examples of discrete behavior in the physical domain (e.g. blackbody radiation). An additional component must be the source of the quantization ... namely measure.

Informativity differs from many of the more speculative models, supported by more than 20 verifiable and verified experimental results across several disciplines. The Heisenberg Uncertainty Principle, for instance, demonstrates the physical significance of fundamental units of measure ([1], Equations (53)-(57)). The Shwartz and Harris experiments ([1], Table 1) demonstrate the physical significance of $\theta_{s i}$, an important constant and prediction of the model. Supporting measurements, each a match to four significant digits, of the quantity, age, density and temperature of the $\mathrm{CMB}$ extend the physical domain to the earliest epoch ([1], Section 3.15). Unlike the Standard Model, there is no need to introduce scope parameters to avoid singularities. Those bounds are precisely defined; for example one such bound where $v<\mathrm{c}$ is

$$
n_{L r}>2 n_{M}
$$


Scope parameters, modifications of the known laws of physics (MOND), inflation theory, quintessence and perturbation theory all become unnecessary patches to a well-defined system of counting physically significant units of fundamental measure.

\subsection{Frameworks}

Framework selection also plays an important role in the success of a model. The term, framework, alludes to the level of detail carried by the terms in our expression nomenclature. Examples include terms such as $G, \hbar, \mathrm{c}, v, t$ and $E$. Frameworks also include operators, such as $+,-, *, /, \sqrt{ }, \sigma$ and $\int$. Poorly chosen frameworks do not invalidate expressions. They establish a level of granularity with which to describe nature in much the same way as precision determines the number of significant digits in a result. Naturally, if detail is lacking, we will not be able to resolve the underlying structure.

Notably, the granularity of the terms $l_{f} m_{f} \theta_{s i}$ and $t_{f}$ are equally important. Writing expressions using a framework that preserves the fundamental measures or counts of them cannot be understated. Consider, for instance, what the expression for escape velocity at the surface of a black hole $r c^{2}=2 G M$ describes (Standard Model). Now consider the same expression when written in a fundamental nomenclature, $n_{L r}>2 n_{M}$ (Informativity). The latter, ideally, presents fewer questions. Consider now, theoretically, if we were to start the argument with the Pythagorean Theorem, derive the expression for escape velocity and then produce the fundamental expression $n_{L r}>2 n_{M}$. This presentation, ideally, would demonstrate a foundation that offers even fewer questions. The level of detail at which our variable nomenclature is defined and the operators used in simplifying those expressions determine how much detail is retained, how effective the model can be.

\subsection{Models}

In modern theory we ask, what is missing from the Standard Model in light of its limitations. For instance, we have not been able to use the Standard Model to solve for a quantum understanding of gravity. The model can provide no explanation for universal expansion or dark matter. Particles have been suggested, but thus far no evidence has been demonstrated nor has the model provided viable candidates. At least one particle prediction is in conflict, the neutrino [4] which is predicted to have no mass under the classic Standard Model. Several other conflicts have been brought to the table, but discussion of these issues introduces lines of thought based on such a complex assortment of axioms, it is difficult to say where the issue is. As is true with all models, these challenges do not mean that a model is inaccurate, only that one or perhaps several axioms are not entirely accurate. At issue, the Standard Model presumes an unbounded physical domain in contradiction of experimental support for a bound to measure ([1], Equation (57)). 
General Relativity builds on top of the Standard Model with important departures. For one, time is treated as an extra dimension along side of length. Mathematically and physically, this is supported, but it is no more valid than describing mass as a dimension. Integration of the physically distinct measures into a single mathematical nomenclature can afford us the ability to apply well-known geometric principles. At the same time, it disguises the details.

A notable example is space-time curvature. Exactly, what is curved-space or time. Or, in light of a fundamental nomenclature, is curvature a differential in the discrete unit counts of space and time? The consideration reveals the issue. Is this not a framework issue? The expressions are accurate, but the details are blurred.

This is not to say that GR hasn't been successful or that any part of GR is incorrect. GR is physically significant, but not applicable throughout the physical domain. Challenges are met at the extreme when applied, for example, to the birth of the universe. It is here that a discrete under-structure is required to define scope, protect from singularities [5] and provide the meaning we are seeking.

Quantum Mechanics is considerably different, a combination of a separable Hilbert space (a.ka. the state space) in conjunction with a complex number of norm 1, the phase factor. Where a given system is considered expressions are developed for each observable as a self-adjoint acting on the state space. Eigenstates of the observables correspond to respective eigenvectors of the operators and the eigenvalue corresponds to the value of the observable for that eigenstate. These mathematical constructs then allow for calculations of the probability of an outcome. Quantum Mechanics can tell us about the probability of an event and the effects of constrained information on a system. Quantum Mechanics can also tell us about events where information is not constrained, but these constructs become less effective at revealing the discrete details measured within the classical domain. While Informativity is able to precisely identify the demarcation point between classical and quantum behavior (e.g. [1], Equation (57)), the two theories do not offer a mathematical bridge between them. In correspondence to the physical data, a two model approach is essential and while quantum mechanics can provide a singular description of phenomena in both domains, the discrete components of classical behavior are incompatible with the mathematical nomenclature needed to describe an information constrained quantum system.

At the cosmological end of the measurement spectrum, $\Lambda \mathrm{CDM}$ has had exceptional success describing mass/energy distribution from the perspective of an inertial frame, but does so through a parameterization with respect to the cosmological constant. The model is challenged to tell a complete story, requiring inflation and quintessence to fill a gap that starts with the earliest epoch. Inflation, in turn, requires faster-than-light expansion, a well-known violation of the laws of physics. But, these issues do not call into question the model, which is highly accurate at describing cosmological mass distribution, hydrogen/helium 
distribution and the expansion of the universe.

Unfortunately, like Quantum Mechanics, the discrete classical elements of physical behavior are lost in the nomenclature. More simply put, $\Lambda \mathrm{CDM}$ is not a quantum model nor does the approach allow for retention of a discrete nomenclature.

Conversely, Informativity's success in resolving the mass distributions is possible only because the fundamental units and there relation allow a calculation of the mass to length frequency bound, which gives us the fundamental mass of the universe ([1], Equation (93)). With that, the mass distributions (i.e. [1] dark mass (Equation (109)), visible (Equation (113)), observable (Equation (110)) and unobserved (Equation (115)) may be resolved. Without this, the physical domain is unbounded necessitating speculative patches such as inflation, quintessence and artificial scope restriction.

LQG takes an approach distinct from the Standard Model, a background independent framework and the quantization of space and time, with a significant investment in Riemannian geometry. The model begins with relativity as a platform and then adds a discrete quantum fabric specifically for length and time. The goal is to integrate gravity into Einstein's initial geometric formulations while at the same time avoiding the nonrenormalizable divergences encountered in quantum field theories. The approach describes space as a grid of interwoven finite loops called spin networks, a field of research known as Spin Foam Theory ([6], Section IX, B). Perhaps one of the more interesting similarities between LQG and Informativity is the idea that gravitons might be the outcome of a semi-classical or weak field limit, clues that may arise from specific mathematical bounds created by the inference of a discrete space-time. This is not to say that Informativity supports the concept of gravitons - quite the opposite; only to say that limits and bounds are important to discrete models in explaining phenomena.

With respect to Informativity, LQG grabs hold of the key ingredients, a discrete underlying fabric with background independence, features that have allowed Informativity to present expressions for some of the most difficult problems in modern theory (e.g. dark energy, expansion, quantum inflation, quantum gravity). LQG is largely hinged on the application of a discrete under-structure on which everything is described, but no semi-classical limit recovering GR has been shown to exist. This calls into question the primary axiom, that a Planck scale space-time ([6], Section I, A) has a corresponding continuum limit as described by GR. Of equal concern is the use of a Hamiltonian constraint ([9], Section II) also in the face of no experimental support [3]. Several other issues, for example the coupling to matter fields of quantum field theory and the renormalization of the graviton have created headwinds [7].

Informativity is able to extend beyond these issues because of its differing axioms. Specifically, Informativity holds that it isn't space-time that is discrete. Measure is discrete, a physically significant property of observation. Secondly, Informativity recognizes $\theta_{s i}$ as a physically significant and measurable value. 
Several experimental results support its significance, for one the Shwartz and Harris experiments on quantum entanglement [8]. Informativity furthers that argument with experimental support in gravitation ([1], Section 3.3), dark energy ([1], Section 3.12), quantum inflation ([1], Section 3.14) and the CMB ([1], Section 3.15).

\subsection{Conclusions}

In this review we are repeatedly reminded that the choice of framework does not affect the accuracy of an expression. One may take the results as described in one framework and translate to a more granular framework revealing the underlying structure. Without a nomenclature defined with respect to the fundamental measures and operators well fitted to manipulating discrete measures, physical expressions are difficult to interpret; the granular details are lost. It is with this foundation that so many models of modern thought have found promise in the face of an unbounded physical domain. It might be proposed that small modifications to existing axioms could provide the scope restrictions that resolve the false outcomes and singularities. But, what does this achieve?

Our analysis, thus far, has barely touched on the complexity of these models and there is an explicit reason for this. Attempts to correct axiom and nomenclature differences effectively translate those works into Informativity. Modifying expressions from one framework to another by changing the nomenclature or underlying axioms fails to accomplish a goal. At best, we are subsuming the successes of other approaches. At worst, where two models differ in axiom, it is difficult to ascertain the accuracy or correctness of a result. Cross model comparisons are questionable, a mixing of truths and non-truths difficult to unravel.

\section{Methods}

\subsection{Fundamental Measures}

Concepts prerequisite to understanding the expressions within will be visited such as measurement bounds, frames of reference and quantum gravity.

As the physical significance of measure is instrumental to our discussion, we present Heisenberg's Uncertainty Principle [9] as applied to the position and momentum of a particle. The expression when reduced to a count $n_{M}$ of mass $m_{f}$ in a system, a count $n_{L r}$ of length $l_{f}$ between an observed target and a center of gravity (i.e. the point from which the weight of the system may be considered to act), and a count $n_{L}$ of $l_{f}$ between the observer and the target describing the target's speed is then ([1], Equation (54))

$$
n_{M} n_{L r} n_{L} \geq l_{f}
$$

Thus, as demonstrated by the Uncertainty Principle, we also find a fundamental unit of length $l_{f}$ to be physically significant, thereby defining a threshold. Using other physical constraints such as the speed of light $c=l_{f} / t_{f}$ this result can be extended to demonstrate the physical significance of time $t_{f}$ and the importance of fundamental mass $m_{f}$ as a countable value of significance. 
The process of measurement quantization is one of converting existing physical descriptions into terms that represent counts of length $l_{f}$ mass $m_{f}$ and time $t_{f}$ It should be noted that while $l_{f}$ and $t_{f}$ each represent a smallest physically significant measure, $m_{f}$ does not. Mass units do play an important role though in describing phenomena, and as such we refer to them as fundamental, which is only meant to say that they are countable and physically significant.

To distinguish the approach from that of Planck's Units [10] [11], we subscript each measure with the letter $f$, which has a historical importance where expressions sometimes mix Planck's units of measure with those of Informativity. There is a correlation between the two and a reason for their difference, but the scope of this paper is confined to the contraction and dilation of measure.

Also note, where the contraction and dilation of measure is commonly discussed, we instead use the phrase, measurement distortion. This is intended only as a short-hand to the longer more commonly used phrase.

Measurement quantization may be described where you consider measuring the length of a stick as a count of some reference. You might take a similar stick, break it into small pieces and then use the smallest of those pieces as your reference. The reference may be used to define a unit of length and with that you can now resolve how many units of the reference are needed to match the length of any object.

Nevertheless, in developing this approach, targets smaller than the reference are encountered. Fractional counts of the reference (i.e., length or time) fail our goal of finding an appropriate reference. As such, we need to continue breaking the stick until a reference is found that is smaller than all targets. We do not need to determine what the reference is or to resolve some understanding of its size. We only need to agree that the existence of a smallest reference is physically significant.

With our reference, we now imagine a mathematical description of a phenomenon that is in length not a whole-unit count of the reference. For instance, consider the hypotenuse of a right triangle, i.e., the side opposite the right angle, the remaining sides being each a count of one unit of the reference. Such a description describes a hypotenuse that is $\sqrt{2}$ units of the reference.

Observation of a fractional measure, in short, fails to comply with the definition of a reference-that all measures can only be a whole-unit count of the reference. The constraint divides the description of phenomena in half, expressions that describe a known and those that describe where information is constrained. Heisenberg's Uncertainty Principle identifies a dividing point.

Note that there is a distinction between measure and its properties where measure is applied.

$O_{1}$ : Recognition of physically significant units of measure does not imply that the target is discrete, only that measure is discrete.

That is to say, the physical significance of discrete units of length does not imply that space is discrete. 
Finally, a discussion of the foundations of measurement quantization may be found by reading "Measurement Quantization Unites Classical and Quantum Physics" [1]. However, the subject of this paper is specific to measurement distortion and how application of measurement quantization leads to verifiable predictions for the entire measurement domain.

\subsection{Gravity}

We begin by refining our understanding of observation in terms of three measures. In contrast to Einstein's presentation of Special Relativity (SR) [12], where expressions describe measurement distortion with respect to an inertial frame, we shall first look at what defines measure.

Consider two points. We may use Figure 2 and the Pythagorean Theorem to describe the distance between them. Specifically,

$$
\begin{gathered}
\left(b_{L}+Q_{L}\right)=\sqrt{a_{L}^{2}+b_{L}^{2}} \\
Q_{L}=\sqrt{1+b_{L}^{2}}-b_{L}
\end{gathered}
$$

and our reference count is always $a_{L}=1$. Then, there is some known count $b_{L}$ of the reference $l_{f}$ that resolves the unknown count, side $c$, between the target and a center of gravity. With $Q_{L}$ the remainder beyond the whole-unit count, we find that the hypotenuse is always the count $b_{L}$ plus a fraction $Q_{L}$ of the reference.

Note that the distance between points $\mathbf{A}$ and $\mathbf{C}$ is described as a count of $I_{f}$ between a target and a center of gravity. This distance may also be described as a count of $l_{f}$ between a target and an observer; the distance to be used depends on whether we are discussing measurement distortion with respect to gravitation $n_{L r}$ (i.e., replace $b_{L}$ with $n_{L r}$ ) or motion $n_{L m}$. Presently, we shall summarize a quantum description of gravity as originally presented in [1].

Multiplying the change ratio $Q_{L} / n_{L r}$ by length $l_{f}$ and dividing by time $t_{f}^{2}$ places the expression in SI units. Multiplying by the speed of light $\mathrm{c}$ and dividing by the scalar constant $S$ adjusts the expression for the expansion of space. Thus,

$$
\frac{Q_{L} l_{f}}{n_{L r} t_{f}^{2}} \frac{c}{S}=\frac{Q_{L} c^{2}}{n_{L r} t_{f} S}=\frac{Q_{L} l_{f} c^{2}}{n_{L r} l_{f} t_{f} S}=\frac{Q_{L} c^{3}}{r S}
$$

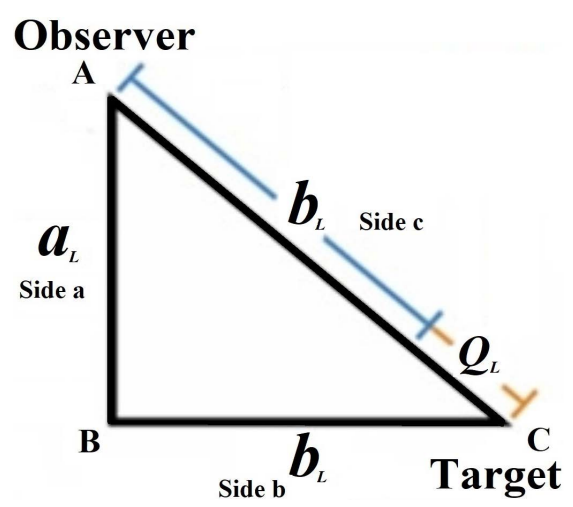

Figure 2. Counts of $l_{f}$ between a target and a center of gravity. 
If the fractional count $Q_{L}$ with respect to a center of gravity $n_{L r}$ is lost at each count of $t_{f}$ then the fractional losses describe gravity in quantum form.

We may set Newton's expression $G / r^{2}$ to be approximately equivalent to better understand their relation;

$$
\begin{gathered}
\frac{Q_{L} c^{3}}{r S} \approx \frac{G}{r^{2}} \\
Q_{L} r c^{3} \approx G S
\end{gathered}
$$

The scalar constant $S$ is central to measurement quantization. Specifically, after converting an expression to counts of the fundamental measures, nearly every physical expression includes this constant. Informativity recognizes and cites several examples of measurement data demonstrating the physical significance of $S$, such as an angular measure specific to the polarization of X-rays in pure Bell states necessary to entangle photons and, as well, half the momentum of a fundamental measure of mass. For this reason, the constant is here on denoted by $\theta_{s i}$ not because it is an angular measure in all situations, but to emphasize the invariant nature of this constant regardless of the frame of reference.

Describing and supporting a complete understanding of $\theta_{s i}$ is beyond the scope of this paper but may be understood with greater detail in the first paper [1]. That said, we shall discuss the importance of frames of reference and how they affect our description of physical phenomena. At this juncture, note that frames of reference determine what units should be associated with $\theta_{s i}$ In some instances, the described phenomenon requires an understanding as an angular measure or momentum, and in others $\theta_{s i}$ has no units at all (i.e., where the expression describes an upper or lower bound to measure). While the first two are commonly apparent, the latter is explored further in Section 3.8.

For now, note that with $\theta_{s i} \mathrm{c}$, and $G$, each of the fundamental measures are resolved ([1], Equations (20)-(22)) such that

$$
\begin{gathered}
l_{f}=\frac{2 G \theta_{s i}}{c^{3}}=\frac{2 \times 6.67408 \times 10^{-11} \times 3.26239}{(299792458)^{3}}=1.61620 \times 10^{-35} \mathrm{~m}, \\
t_{f}=\frac{l_{f}}{c}=\frac{2 G \theta_{s i}}{c^{4}}=\frac{2 \times 6.67408 \times 10^{-11} \times 3.26239}{(299792458)^{4}}=5.39106 \times 10^{-44} \mathrm{~s}, \\
m_{f}=t_{f} \frac{c^{3}}{G}=\frac{2 \theta_{s i}}{c}=\frac{2 \times 3.26239}{299792458}=2.17643 \times 10^{-8} \mathrm{~kg} .
\end{gathered}
$$

The three measures may be combined to reveal their relation as

$$
l_{f} m_{f}=2 \theta_{s i} t_{f}
$$

which we formally recognize as the fundamental expression. Notably, where $\lim _{r=\infty} f\left(Q_{L} n_{L r}\right)=1 / 2$ ([1], Appendix A), the expression is appropriate where gravity is not a consideration. Where gravity is applicable, the expression is written as

$$
l_{f} m_{f}=\frac{\theta_{s i} t_{f}}{Q_{L} n_{L r}} .
$$


Measurement quantization is a unique approach that allows for a quantum description of measure with respect to motion and gravitational fields. Nonetheless, it is difficult if not entirely inappropriate to compare Einstein's work on the contraction and dilation of measure to that of Informativity. For one, the Informativity differential $Q_{L} n_{L r}$ describes a new form of measurement distortion, a specific outcome due to measurement bounds of the whole-unit count of a reference. The approach may be extended to measurement distortion for a static target as well as one in motion or in a gravitational field.

In contrast, the effects described by Relativity in a gravitational field are premised on an understanding of space that differs from Informativity. GR introduces the idea of a curved space-time. The implication is that the reference measures $l_{f} m_{f}$ and $t_{f}$ vary in the local frame with respect to a center of gravity. Informativity is premised on the idea that the reference measures are simply references, and as such any description of space (i.e., $I_{f}$ ) cannot include properties that vary such as "curvature".

These points of view are incompatible, although the theories are not. Both Informativity and Relativity each make predictions that match our best measurement data but do so with an important difference. When discussing measurement distortion, Informativity recognizes that it is the count of the reference that varies in a gravitational field, not the reference. Furthermore, instrumental in describing curvature is the fact that the measure $Q_{L}$ is less than the reference.

\subsection{Nomenclature}

Let us take this moment to discuss the nomenclature used to describe counts of the fundamental measures. Where all counts are denoted with the symbol $n$, each measure is recognized by a corresponding capitalized subscript, $L$ for length, $M$ for mass and $T$ for time. To avoid confusion between length descriptions of motion and those of gravitational fields, we append a subscript $r$ (i.e., $n_{L r}$ ) when describing a count of $l_{f}$ between an observed target and a center of gravity. Similarly, we append a subscript $m$ (i.e., $n_{L m}$ ) when describing a change in the count of $l_{f}$ with respect to a target in motion to the observer.

When describing contraction and dilation expressions, these subscripts are understood to be fundamental unless otherwise noted. They are then followed by either a subscript $l$ indicating measure in the "local" inertial frame (i.e., $n_{L l}$ ) or a subscript $o$ indicating measure of the "observed" frame (i.e., $n_{\text {Lo }}$ ).

The nomenclature, in part, diverges from modern theory in describing relativistic phenomena. Let us consider the classic example of a train and an observer beside the tracks. When describing the length contraction of a ruler held steady with respect to the motion of the train as observed by our observer, length is denoted by the term on the left $l_{1}$ where

$$
l_{1}=l_{2}\left(1-\frac{v^{2}}{c^{2}}\right)^{1 / 2} \text {. }
$$


Conversely, when describing time dilation, for example, a clock held steady with respect to the motion of the train as observed by our observer, the measure being observed is now denoted by the term on the right $t_{2}$ where

$$
t_{1}=\frac{t_{2}}{\left(1-\frac{v^{2}}{c^{2}}\right)^{1 / 2}} .
$$

In each instance, we are describing an object held steady with respect to the motion of the train as observed from the side of the tracks. However, for length, $l_{1}$ represents the length of the object on the train and for time, $t_{1}$ represents elapsed time in the local frame of the observer. With this approach, one describes length as contracted whereas time is dilated. This becomes very confusing when we introduce counts of the fundamental measures. Both a count of length units representing the ruler and a count of time units elapsed on the clock contract; where both the ruler and the clock being observed are on the train, both counts are less.

We continue to use the traditional terms contraction and dilation, but we always designate the observed measure with the subscript $o$ and when possible to place this term on the left when presenting contraction and dilation expressions. Measures with subscript $l$ always represent a measure in the "local" or rest frame of the observer and typically are found on the right side of the equality. For clarity, the length and time expressions are then consistently written from the observer's point-of-view as

$$
\begin{aligned}
& l_{o}=l_{l}\left(1-\frac{v^{2}}{c^{2}}\right)^{1 / 2}, \\
& t_{o}=t_{l}\left(1-\frac{v^{2}}{c^{2}}\right)^{1 / 2} .
\end{aligned}
$$

Lastly, we commonly use the terms quantum and quantized throughout this paper. Neither should be understood as having a relation with respect to quantum mechanics. Rather, the term quantum is intended to mean small as in a few tens, hundreds or thousands of fundamental units of measure. The term quantized is intended to mean that expressions are composed of terms that are whole-unit counts of the fundamental units and that those units are physically significant.

A quantized expression inherits qualities that are immensely valuable in our effort to describe nature. For one, quantized expressions are defined for the entire measurement domain. Second, quantized expressions are nondimensionalized. Nondimensionalization is not in itself a valuable endeavor but demonstrating that all phenomena may be expressed entirely with nondimensionalized whole-unit counts of the fundamental measures contributes to a new understanding of measure that is finite and discrete. 


\section{Results}

\subsection{Measurement Distortion with Respect to Motion}

In contrast to the traditional approach to describe the contraction and dilation of measure, we seek to describe length using our understanding of measurement bounds and Pythagoras' Theorem,

$$
a^{2}+b^{2}=c^{2} .
$$

We begin by defining terms that account for all possible values that a count of the fundamental measures may take. For example, a count of $l_{f}$ may take any value from 0 to $1.85492 \times 10^{43}$ units/s (i.e., $c / I_{f}$ ). Hence, describing each count range, there is then a maximum $a_{\max }$ and a minimum $b_{\min }$ such that

$$
a_{\max }^{2}+b_{\min }^{2}=c_{\text {bound }}^{2} .
$$

Next, we recognize that each bound may be described as a count $n$ of a fundamental measure. Therefore, where $n_{\text {bound }}$ constrains $n_{\max }$ and $n_{\min }$, we may introduce the variable terms $a$ and $b$ to allow sides $\mathbf{a}$ and $\mathbf{b}$ to vary with respect to their count bounds. The construct then allows a to vary from 0 to $n_{\max }$ and $b$ to vary from 0 to $n_{m i n}$,

$$
\left(a n_{\max }\right)^{2}+\left(b n_{\min }\right)^{2}=n_{\text {bound }}^{2}
$$

There exist only two basic operators we may use to constrain $n_{\text {bound }}$. Unfortunately, the product cannot resolve a fixed interval that is consistent for both ranges,

$$
\begin{aligned}
& n_{\text {bound }}=\left(\left(0 n_{\text {max }}\right)^{2}+\left(n_{\text {min }} n_{\text {min }}\right)^{2}\right)^{1 / 2}=n_{\text {min }}, \\
& n_{\text {bound }}=\left(\left(n_{\text {max }} n_{\text {max }}\right)^{2}+\left(0 n_{\text {min }}\right)^{2}\right)^{1 / 2}=n_{\text {max }} .
\end{aligned}
$$

However, when using a divisor relation

$$
\left(\frac{a}{n_{\max }}\right)^{2}+\left(\frac{b}{n_{\min }}\right)^{2}=n_{\text {bound }}^{2},
$$

then the corresponding intervals may be proportionally constrained,

$$
\begin{aligned}
& n_{\text {bound }}=\left(\left(\frac{0}{n_{\text {max }}}\right)^{2}+\left(\frac{n_{\text {min }}}{n_{\text {min }}}\right)^{2}\right)^{1 / 2}=1, \\
& n_{\text {bound }}=\left(\left(\frac{n_{\text {max }}}{n_{\text {max }}}\right)^{2}+\left(\frac{0}{n_{\text {min }}}\right)^{2}\right)^{1 / 2}=1 .
\end{aligned}
$$

We anchor each range at their shared interval endpoint. Variable a may take on any value between 0 and $n_{\max }$ to resolve the corresponding value for $b$,

$$
\left(\frac{a}{n_{\max }}\right)^{2}+\left(\frac{b}{n_{\min }}\right)^{2}=1 \text {. }
$$

As such, we recognize that all phenomena may be described either with or as a 
modified form of this expression (i.e., gravity, mass, expansion). We refer to such expressions as unity expressions.

By way of example, we use light to describe length. Replace $n_{\max }$ where $n_{L c}$ is the maximum count of $l_{f}$ traveled by light in a second and replace $n_{\min }$ where $n_{L I}$ is the corresponding minimum count of $l_{f}$ both of which are given in the local frame,

$$
\left(\frac{a}{n_{L c}}\right)^{2}+\left(\frac{b}{n_{L l}}\right)^{2}=1 .
$$

We now introduce the associated variables where $n_{L m}$ is a count of $l_{f}$ between 0 and $n_{L c}$ describing the relative change (i.e., motion) of the observed frame. In addition, $n_{L o}$ is a count of $l_{f}$ between 0 and $n_{L I}$ describing the length in the observed frame. The time interval selected is irrelevant so long as it is consistent for all terms. The assignments are constrained where $n_{L o}$ must be a minimum and therefore can only describe the observed fundamental length. In practice, any length may be chosen but only because all values are translated accordingly. Likewise, only the relative change $n_{L m}$ of the observed frame can vary from 0 to $n_{L o}$

$$
\left(\frac{n_{L m}}{n_{L c}}\right)^{2}+\left(\frac{n_{L o}}{n_{L l}}\right)^{2}=1 .
$$

Using counts of $l_{f}$ draws attention to the mathematical qualities of the Pythagorean relation. The expression is more commonly arranged in the form

$$
\begin{gathered}
\frac{n_{L o}^{2}}{n_{L l}^{2}}=\left(1-\frac{n_{L m}^{2}}{n_{L c}^{2}}\right), \\
n_{L o}=n_{L l}\left(1-\frac{n_{L m}^{2}}{n_{L c}^{2}}\right)^{1 / 2} .
\end{gathered}
$$

Finally, by translating the count terms to SI units with the ratio $l_{f} t_{f}$ we recognize that the speed parameter $\beta^{2}$ is

$$
\frac{n_{L m}^{2}}{n_{L c}^{2}}=\left(\frac{n_{L m} l_{f}}{t_{f}}\right)^{2}\left(\frac{t_{f}}{n_{L c} l_{f}}\right)^{2}=\frac{v^{2}}{c^{2}} .
$$

By substitution, the corresponding expressions for time and mass are

$$
\begin{gathered}
n_{T o}=n_{T l}\left(1-\frac{n_{L m}^{2}}{n_{L c}^{2}}\right)^{1 / 2}, \\
n_{M o}=n_{M l} /\left(1-\frac{n_{L m}^{2}}{n_{L c}^{2}}\right)^{1 / 2} .
\end{gathered}
$$

One typically measures the numerator (velocity) as a rate of change in position and length contraction as a function of that change with respect to the upper bound $n_{L C}$ In contrast, contraction may be described entirely as a count $n_{L m}$ 
of $l_{f}$ reflecting the ratio of the target's change in length count to the count bound $n_{L c}$ Thus, the introduction of $l_{f} / t_{f}$ with respect to these counts is an unnecessary and superfluous translation that does not contribute to describing the contraction or dilation of measure. We may then observe that:

$\mathrm{O}_{2}$ : The best suited description of the contraction and dilation of measure is a count change.

To avoid confusion, we continue to use the terms contraction and dilation, but forthwith it is understood that we are talking about a count difference between two inertial frames. For consistency of nomenclature, we always identify counts as relative to the local inertial frame $n_{L I}$ (i.e., the observer) or the observed inertial frame $n_{L o}$ (i.e., the target).

To present length contraction in SI units, multiply by $l_{f}$ (i.e., $l_{o}=n_{L o} l_{f} l_{l}=n_{L l} l_{f}$ ), replace the speed parameter where $n_{L m}^{2} / n_{L c}^{2}=v^{2} / c^{2}$ and consolidate such that Equation (31) becomes

$$
\begin{gathered}
n_{L o} l_{f}=n_{L l} l_{f}\left(1-\frac{n_{L m}^{2}}{n_{L c}^{2}}\right)^{1 / 2}, \\
l_{o}=l_{l}\left(1-\frac{v^{2}}{c^{2}}\right)^{1 / 2} .
\end{gathered}
$$

The final expression now mirrors that of Einstein's. Note well, our detour from the modern approach demonstrates new details that we may recognize as a required outcome of bound measure.

In addition, measurement quantization introduces physically significant bounds to measure that are not properties of the observed phenomenon. A good example is the measure $Q_{L}$ described at the outset of Section 2.2. If $Q_{L}$ describes a physically significant phenomenon as an outcome of measure-gravity-this does not imply that the space around a mass varies in length.

$\mathrm{O}_{3}$ : Measurement quantization does not imply that space is discrete, only that measure is discrete.

Lastly, we should note that the count bounds of the fundamental measures-length, mass, and time-are the same when defined relative to a given measure:

$$
\begin{gathered}
n_{L}=2.99792458 \times 10^{8} / l_{f}=1.85492 \times 10^{43} \text { units } / \mathrm{s}, \\
n_{M}=4.0371111 \times 10^{35} / m_{f}=1.85492 \times 10^{43} \text { units } / \mathrm{s}, \\
n_{T}=1 / t_{f}=1.85492 \times 10^{43} \text { units } / \mathrm{s} .
\end{gathered}
$$

As such, one may interchange the upper bound counts as may apply to a measurement of the target.

$$
n_{L}=n_{T}=n_{M} .
$$

While this can be useful, the correlation brings to our attention a principle. Identified measurement bounds can be used to interchange units or constrain an expression facilitating a solution. 


\subsection{Understanding Immeasurable Space}

When developing expressions that describe the contraction and dilation of measure in a gravitational field, one may use escape velocity to correlate the relativistic effects of motion to that of gravity. An abundance of experimental results confirms this approach.

Conversely, measurement quantization already recognizes gravitation as the loss of fractional counts $Q_{L}$ of the reference $l_{f}$ with respect to the square of each instant in time $t_{f}$ That is, measurement quantization describes gravitation as motion. We may use that understanding to present a quantum model of measure in a gravitational field. But, before we begin, let us establish a better understanding of $Q_{L}$, the immeasurable space.

As depicted in Figure 3, we may provide a one-to-one correlation between the immeasurable space and the distance to a center of gravity $n_{L r}$. Here, side a is $l_{l}=$ $n_{L l} l_{f}$ (the reference $n_{L l}=1$ ), side $\mathrm{b}$ is a known distance $l_{r}=n_{L I} l_{f}$ and side $\mathrm{c}$ is the unknown distance from a point to a center of gravity $\left(n_{L r}+Q_{L}\right) I_{\dot{p}}$ then,

$$
\begin{gathered}
l_{l}^{2}+l_{r}^{2}=\left(Q_{L}+l_{r}\right)^{2}, \\
\left(n_{L l}^{2}+n_{L r}^{2}\right) l_{f}^{2}=\left(Q_{L}+n_{L r}\right)^{2} l_{f}^{2}, \\
n_{L l}^{2}+n_{L r}^{2}=Q_{L}^{2}+2 Q_{L} n_{L r}+n_{L r}^{2}, \\
1=Q_{L}^{2}+2 Q_{L} n_{L r}, \\
n_{L r}=\frac{1-Q_{L}^{2}}{2 Q_{L}}=\frac{1-Q_{L}^{2}}{2 Q_{L}}=\frac{1}{2}\left(\frac{1-Q_{L}^{2}}{Q_{L}}\right), \\
n_{L r}=\frac{1}{2}\left(\frac{1}{Q_{L}}-Q_{L}\right) .
\end{gathered}
$$

One might ask, if the measure of space $l_{f}$ does not change near a mass yet the unit count does, then what accounts for the "missing space" with regard to a location where gravity is greater?

The missing space nearer a mass is still there and has been identified with the count term $Q_{L}$ but it cannot be measured because it is smaller than the reference measure $l_{f}$ We have described this space with the Informativity differential; it is what describes the phenomenon of gravitation.

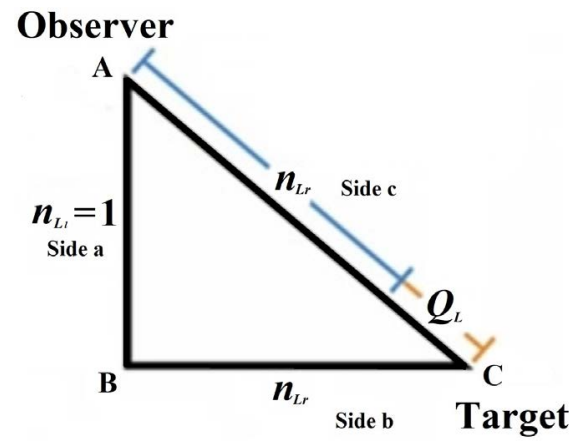

Figure 3. Count of distance measures between an observer and a center of gravity. 
We should clarify that space (i.e., the measure of whole-unit counts of $l_{f}$ ) is geometrically flat around a mass. Nevertheless, it is the physical significance of upper and lower bounds to measure that produce the phenomenon we recognize as gravity. Whereas modern theory recognizes a curved space $l_{r}$, measurement quantization breaks down its component terms $l_{r}=n_{L l} l_{f}$ to demonstrate that it is not space $l_{f}$ that varies, but the count of the invariant and physically significant fundamental measures that vary with each increment of time $t_{f}$ That variation is appropriately labeled as a count differential as described by the Informativity differential $Q_{L} n_{L r}$

We may also arrange the expression to demonstrate that

$$
\begin{gathered}
\frac{Q_{L}}{2}+n_{L r}=\frac{1}{2}\left(\frac{1}{Q_{L}}\right), \\
\lim _{n_{L r} \rightarrow \infty}\left(\frac{Q_{L}^{2}}{2}+Q_{L} n_{L r}\right)=\frac{1}{2} .
\end{gathered}
$$

When considering a large distance, the left term drops out giving us the abbreviated form. The Informativity differential $Q_{L} n_{L r}$ is difficult to work with and for this reason the term is often incorporated into Informativity expressions at either the quantum $\left(\lim n_{L r=1} f\left(Q_{L} n_{L r}\right)=\sqrt{2}-1\right)$ or cosmological limit $\left(\lim n_{L r=\infty} f\left(Q_{L} n_{L r}\right)=1 / 2\right)([1]$, Appendix A) depending on the relative distance of the phenomenon being observed.

With this we may then present a quantum description of gravity as the loss of $Q_{L}$ at every instant in time $t_{f}$ Specifically, it is not that space is discrete. Rather, measure is discrete and its physical significance may be demonstrated with Heisenberg's Uncertainty Principle, Equation (4). When an immeasurable distance is physically significant yet less than the reference, then it is conjectured that matter behaves as though the immeasurable distance is discarded at each instant in time $t_{f}$ With this, gravity $\left(G / r^{2}\right)$ may be described as the product of the change in position with respect to a center of gravity $Q_{L} / n_{L r}$ converted to SI units $l_{f} / t_{f}^{2}$ and multiplied by the expansion of space.

Note also that universal expansion is defined such that the leading edge of the knowable universe will always move at the speed of light with respect to the inertial frame of an observer at that edge. The radial rate of universal expansion is initially the unknown value $\theta_{s i}$. With this we describe gravity as a product of the motion associated with the discarded distance $Q_{L} l_{f} / n_{L r} t_{f}^{2}$ multiplied by the rate of linear change (i.e. the ratio of the upper bound $\mathrm{c}$ to the expansion $\theta_{s i}$ ); that is $c / \theta_{s i}$ such that

$$
\frac{Q_{L}}{n_{L r}} \frac{l_{f}}{t_{f}^{2}} \frac{c}{\theta_{s i}} .
$$

The correlation of $\theta_{s i}$ to the Shwartz and Harris experiments is then realized as half the momentum of a fundamental unit of mass $m_{f}$ and the angle in radians that subtends a segment with an arc length of $\hbar$ meters. The descriptions collec- 
tively are shown to have been measured with respect to the polarization of $\mathrm{X}$-rays in specific maximal Bell states necessary to entangle light as assessed in Table 1.

Further details are described in the first paper ([1], Section 3.1) showing that $\theta_{s i}=3.26239$ radians [8]. With this a distance sensitive correlation may be expressed as

$$
\frac{Q_{L} l_{f}}{n_{L r} t_{f}^{2}} \frac{c}{\theta_{s i}}=\frac{Q_{L} c^{2}}{n_{L r} t_{f} \theta_{s i}}=\frac{Q_{L} l_{f} c^{2}}{n_{L r} l_{f} t_{f} \theta_{s i}}=\frac{Q_{L} c^{3}}{r \theta_{s i}} \approx \frac{G}{r^{2}} .
$$

The small difference between the two ratios is a reflection of the precision between the quantum model of Informativity in comparison to Newton's presentation. Newton's expression does not include the distortion effect described by the Informativity differential $Q_{L} n_{L r}$ as numerically assessed in Table 2.

By example, we may calculate $Q_{L}$ at a distance of 1 meter. Using Equation (10) for $l_{f}$ the inverse gives us a count of $l_{f}$ such that side $b=6.18735 \times 10^{34}$ units of $l_{f}$ in one meter. In that side $c=\left(1+b^{2}\right)^{1 / 2}$, then

$$
\begin{gathered}
Q_{L}=c-b=\sqrt{1+b^{2}}-b, \\
Q_{L}=\sqrt{1+\left(6.18735 \times 10^{34}\right)^{2}}-6.18735 \times 10^{34}=8.08100 \times 10^{-36}
\end{gathered}
$$

As distance increases, $Q_{L} n_{L r}$ decreases proportionally as assessed in Table 3.

For distances where the difference from $Q_{L} n_{L r}=1 / 2$ is inconsequential, the expression may be reduced such that

Table 1. Angle setting in radians of the $\mathbf{k}$ vectors of the pump, signal and idler for maximally entangled states at the degenerate frequency with corresponding Shwartz and Harris values (Reference [8]).

\begin{tabular}{cccc}
\hline \multirow{2}{*}{ Bell's State } & $\theta_{p}$ k vector angle \\
\cline { 2 - 4 } & $\theta_{s}$ & $\theta_{i}$ \\
\hline$\left(\left|H_{s}, V_{i}\right\rangle+\left|V_{s}, H_{i}\right\rangle\right) / \sqrt{2}$ & $\left(I_{f} \mathcal{c}^{3} / 2 G\right)-\pi(0.1208)$ & $\pi-\left(I_{f} \mathcal{c}^{3} / 2 G\right)(-0.1208)$ & $\pi-\left(I_{f} \mathcal{c}^{3} / 2 G\right)(-0.1208)$ \\
& $2 \pi-\left(I_{f} \mathcal{c}^{3} / 2 G\right)(3.02079)$ & $\left(I_{f} \mathcal{c}^{2} / 2 G\right)(3.26239)$ & $\left(I_{f} \mathcal{c}^{3} / 2 G\right)(3.26239)$ \\
\hline
\end{tabular}

Table 2. Informativity difference from $G / r^{2}$.

\begin{tabular}{ccccccc}
\hline & \multicolumn{7}{c}{ Difference in $G / r^{2}$} \\
\cline { 2 - 6 } & $50 I_{f}$ & $150 I_{f}$ & $300 I_{f}$ & $500 I_{f}$ & $1000 I_{f}$ & $2248 I_{f}$ \\
\hline Difference & $0.01000 \%$ & $0.00111 \%$ & $0.00028 \%$ & $0.00010 \%$ & $0.00003 \%$ & $0.00000 \%$ \\
\hline
\end{tabular}

Table 3. Calculation of the informativity differential with increasing distance.

\begin{tabular}{cccccc}
\hline & \multicolumn{5}{c}{ Distance } \\
\cline { 2 - 6 } & $50 I_{f}$ & $2000 I_{f}$ & $1 \mathrm{~m}$ & $1 \mathrm{ly}$ & $13.799 \mathrm{bly}$ \\
\hline$Q_{L}$ & $9.99900 \times 10^{-3}$ & $2.50000 \times 10^{-4}$ & $8.08100 \times 10^{-36}$ & $8.54747 \times 10^{-52}$ & $6.19427 \times 10^{-62}$ \\
$1 / 2-Q_{L} n_{L r}$ & $4.99900 \times 10^{-5}$ & $3.12500 \times 10^{-8}$ & $3.26512 \times 10^{-71}$ & $3.65296 \times 10^{-103}$ & $1.91845 \times 10^{-123}$ \\
\hline
\end{tabular}




$$
G \approx \frac{Q_{L} r^{2} c^{3}}{r \theta_{s i}} \approx \frac{Q_{L} n_{L r} l_{f} c^{3}}{\theta_{s i}}=\frac{l_{f} c^{3}}{2 \theta_{s i}} .
$$

The expression may be further reduced when recognizing the following transform. Multiply $c^{3} / G$ by Planck's expression for time $t_{p}=\left(\hbar G / c^{5}\right)^{1 / 2}$, then we get Planck's expression for mass

$$
m_{p}=\frac{c^{3}}{G}\left(\frac{\hbar G}{c^{5}}\right)^{1 / 2}=\left(\frac{c^{6}}{G^{2}} \frac{\hbar G}{c^{5}}\right)^{1 / 2}=\left(\frac{\hbar c}{G}\right)^{1 / 2} .
$$

Thus Equation (53) when organized as $c^{3} / G=2 \theta_{s i} / l_{f}$ and multiplied by $t_{f}$ describes fundamental mass ([1], Equation (11)),

$$
m_{f}=t_{f} \frac{c^{3}}{G}=t_{f} \frac{2 \theta_{s i}}{l_{f}}
$$

which allows us to reduce $G$ such that

$$
G=\frac{l_{f} c^{3}}{2 \theta_{s i}}=c^{3} \frac{l_{f}}{2 \theta_{s i}}=c^{3} \frac{t_{f}}{m_{f}} .
$$

Notably, Equation (55) provides the fundamental expression $l_{f} m_{f}=2 \theta_{s i} t_{f}$ cited in Equation (13).

Also note that the Planck and fundamental masses are not value equivalent. The later incorporates time. The Informativity expression $t_{f}=G \theta_{s i} / Q_{L} n_{L r} c^{4}=$ $5.39106 \times 10^{-44} \mathrm{~s}$ includes the effects of the Informativity differential as described in Table 2. As such, we find that the two expressions:

- $m_{p}=(\hbar c / G)^{1 / 2}=2.176470(51) \times 10^{-8} \mathrm{~kg}[11]$;

- $m_{f}=t_{f} c^{3} / G=2.17643 \times 10^{-8} \mathrm{~kg}$

differ by this effect, $0.4 \times 10^{-12} \mathrm{~kg}$.

To clarify, the CODATA value for $m_{p}$ is calculated as an outcome of interactions measured in relation to the calculation of $\hbar$ and $G$, mixing the quantum interactions typically used in the measure of $\hbar$ with the macroscopic measure of $G$. Conversely, the Informativity calculation corresponds to measurement data subject to variation in $G$ and $t_{f}$ where remaining values are fixed in each inertial frame. In that $G$ is measured macroscopically, $Q_{L} n_{L r}$ (i.e. $t_{f}=\theta_{s i} G / Q_{L} n_{L I} c^{4}$ ) is taken at its upper limit of $1 / 2$ exposing the effects of the Informativity differential which we see as a difference between $m_{f}$ and $m_{p}$.

\subsection{Distortion of Measure with Respect to Gravity}

Recognizing that the Informativity differential is a description of gravitation as motion, we may write expressions with respect to mass as easily as those with respect to motion, thus completing the quantized expressions with respect to a gravitational field. Starting with the expression above, then

$$
\begin{gathered}
G=c^{3} \frac{t_{f}}{m_{f}}=c^{2} \frac{l_{f}}{t_{f}} \frac{t_{f}}{m_{f}}=c^{2} \frac{l_{f}}{m_{f}}, \\
2 c^{2}=2 \frac{G m_{f}}{l_{f}},
\end{gathered}
$$




$$
\sqrt{2} c=\left(\frac{2 G m_{f}}{l_{f}}\right)^{1 / 2} .
$$

The expression correlates the numerical change in length count $\sqrt{2} c$ with respect to a gravitational field for the specific instance where Pythagorean sides a and $\mathbf{b}$ are each a count of 1 (i.e., $\left.\left(1^{2}+1^{2}\right) 1 / 2=\sqrt{2}\right)$. At this point, we focus only on what in modern theory is understood as the measurement distortion associated with gravitation; we do not consider the effects of the Informativity differential. In essence, we are taking the Informativity differential at its cosmological limit.

To generalize the expression and encompass velocity as corresponds to any mass $n_{M} m_{f}$ with respect to any radial distance $n_{L l} l_{f}$ from a center of gravity, we introduce the respective count terms associated with each measure. This is more complex for the left term as the $\sqrt{2}$ is a Pythagorean result which must be expanded. We attend to this first. To begin, we recognize from Figure 2 that

$$
n_{L l}^{2}+n_{L r}^{2}=n_{L m}^{2} \text {, }
$$

and substitute $\sqrt{2}$ with the "generalized" count values $\left(n_{L l}^{2}+n_{L r}^{2}\right)^{1 / 2}$ with $n_{L m}$ being the target's change in position (i.e., motion $n_{L m}$ ),

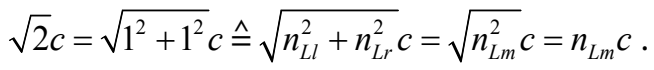

Recalling also Equation (26),

$$
\frac{n_{L o}^{2}}{n_{L l}^{2}}+\frac{n_{L m}^{2}}{n_{L c}^{2}}=1
$$

we recognize that the measure of side $\mathbf{b}$ when cast in SI units implies a time reference. With Equation (40), we recognize the value-equivalent relation $n_{L c}=n_{T c}$ and make the substitution of units in the denominator. Then, for a given count $n_{L m}$ of $l_{f}$ per unit of $t_{f}$ we resolve the associated gravitational motion,

$$
n_{L m}^{2} c^{2}=\frac{n_{L m}^{2}}{n_{L c}^{2}} \frac{l_{f}^{2}}{t_{f}^{2}}=\frac{n_{L m}^{2} l_{f}^{2}}{n_{T c}^{2} t_{f}^{2}}=\frac{l_{m}^{2}}{t_{c}^{2}}=v^{2} .
$$

The relation follows the form $c=l_{f} / t_{f}\left(n_{L}=n_{T}\right)$ and differs where $n_{L m}<n_{T c}$ Thus $v<c$ (i.e. $n_{L m} \mathrm{c}$ is a fractional representation with respect to the speed of light). With $v=n_{L m} c \hat{=} \sqrt{2} c$, we may then generalize the left portion of Equation (59) as the motion associated with this expression,

$$
v=\left(\frac{2 G m_{f}}{l_{f}}\right)^{1 / 2} .
$$

Now we generalize the right portion of the expression for arbitrary mass $m=$ $n_{M} m_{f}$ and gravitational radius $r=n_{L r} l_{f}$ This is not a substitution. The expression presently considers the quantum case, a description of motion in terms of fundamental units. To generalize the expression, we introduce a count $n_{M}$ of $m_{f}$ and a count $n_{L r}$ of $l_{f}$ for the two fundamental units of measure. A generalization is permissible only if the introduced variation is reflected in the left portion of the 
expression $v=I_{m} / t_{c}$ With $G=t_{f} c^{3} / m_{f}$ from Equation (56), then the gravitational parameter is

$$
\begin{gathered}
v^{2}=\frac{2 G n_{M} m_{f}}{n_{L r} l_{f}}=n_{M} m_{f} \frac{2}{n_{L r} l_{f}} \frac{t_{f} c^{3}}{m_{f}}, \\
v^{2}=\frac{2 n_{M} t_{f} c^{3}}{n_{L r} l_{f}}=\frac{2 n_{M} c^{3}}{n_{L r} c}=\frac{2 n_{M} c^{2}}{n_{L r}}, \\
\frac{v^{2}}{c^{2}}=2 \frac{n_{M}}{n_{L r}} .
\end{gathered}
$$

As Equation (32) demonstrates that $v^{2} / c^{2}=n_{L m}^{2} / n_{L c}^{2}$ along with $V^{2} / \mathrm{c}^{2}=$ $2 n_{M} / n_{L r}$, then the gravitational length contraction with respect to the upper bound $n_{L C}$ is

$$
\begin{gathered}
\frac{n_{L m}^{2}}{n_{L c}^{2}}=2 \frac{n_{M}}{n_{L r}}, \\
n_{L m}=n_{L c}\left(2 \frac{n_{M}}{n_{L r}}\right)^{1 / 2} .
\end{gathered}
$$

Note that the expression describes only the distorting effects of the gravitational measurement but should also incorporate those of the Informativity differential where we do not take the limit $Q_{L} n_{L r}=1 / 2$. Accounting for the distorting effects of both measurements, then

$$
\begin{gathered}
n_{L m}=n_{L c}\left(\frac{n_{M}}{Q_{L} n_{L r}^{2}}\right)^{1 / 2}, \\
l_{m}=l_{c}\left(\frac{n_{M}}{Q_{L} n_{L r}^{2}}\right)^{1 / 2} .
\end{gathered}
$$

If we then translate this expression to SI units where $I_{m}=n_{L m} l_{f}$ and $l_{c}=n_{L c} l_{f}$ we find it is inside-out. That is, the change in length count $I_{m}$ for the target is not arbitrary but rather the length count associated with the upper frequency bound, as measured with light in a given time frame. For example, the term $I_{c}$ represents the upper bound in the example range $(0: 1]$ whereas the gravitational parameter $\beta=2 n_{M} / n_{L r}$ represents the variable that is also constrained to the range $(0: 1)$. To reverse the behavior of

$$
l_{c} \sqrt{\beta},
$$

we need to invert the values for $\beta$ in the same range, the inverse expression being

$$
l_{m} \sqrt{1-\beta} \text {. }
$$

This was already known from Equation (67) where $\beta=v^{2} / \mathrm{c}^{2}=2 n_{M} / n_{L r}$. The gravitational parameter may then be applied directly to the newly developed quantum laws of motion, Equations (31), (33), (34).

$$
t_{o}=t_{l}\left(1-2 \frac{n_{M}}{n_{L r}}\right)^{1 / 2},
$$




$$
\begin{gathered}
l_{o}=l_{l}\left(1-2 \frac{n_{M}}{n_{L r}}\right)^{1 / 2}, \\
m_{o}=m_{l} /\left(1-2 \frac{n_{M}}{n_{L r}}\right)^{1 / 2} .
\end{gathered}
$$

Note that as Equation (75) describes measurement distortion as a function of motion, the principle of equivalence is no longer a required axiom. The gravitational distortion of measure is properly described as a geometric property of change between two inertial frames, an outcome of measurement bounds to the physically significant quantization of measure. As such, this description now serves to confirm that the motion that describes gravity and the motion known in classical theory are equivalent. Recognition of a principle of equivalence and the universality of free fall in a gravitational field are no longer axioms but predictions of measurement quantization.

While Einstein disliked the concept of relativistic mass [13], measurement quantization describes the gravitational distortion of measure with defined values for the entire measurement domain. That is, there is an upper bound to mass density and as such there are no undefined results for relativistic mass. That bound is found to be

$$
\begin{gathered}
v=\left(\frac{2 G M}{r}\right)^{1 / 2}, \\
c>\left(\frac{2}{r} \frac{Q_{L} r c^{3}}{\theta_{s i}} \frac{n_{M} \theta_{s i}}{Q_{L} n_{L r} c}\right)^{1 / 2}>\left(\frac{2 n_{M} c^{2}}{n_{L r}}\right)^{1 / 2}, \\
n_{L r}>2 n_{M} .
\end{gathered}
$$

In other words,

$$
2 \frac{n_{M}}{n_{L r}}<1 .
$$

Matter may not have a density of more than two fundamental units of mass $m_{f}$ per fundamental unit of length $l_{f}$ of the gravitational radius considered. Doing so implies a relative count ratio greater than the speed of light violating the count bound $n_{L m}=1.85492 \times 10^{43}$ units/s. The term matter is conjectured to describe baryonic matter. The conjecture stems from our initial definition of $m_{f}$ as a physically significant countable unit of mass. While $m_{f}$ does not describe the smallest example of baryonic mass, the expression above does describe an upper bound to its density.

Note also that $n_{M} \neq 0$ and as such Equations (78)-(80) cannot be $\geq$ expressions. Such a description negates the correlation between length and mass invalidating the relation and detaching the description from the phenomenon it is describing.

Finally, note that all expressions regarding measurement distortion, including those that describe count differentials within a gravitational field, Equation (75), may take the form of the Pythagorean Theorem, $a^{2}+b^{2}=c^{2}$, and as such, length 
contraction may also be presented as

$$
n_{L o}^{2} n_{L r}+2 n_{L l}^{2} n_{M}=n_{L l}^{2} n_{L r} .
$$

We bring attention to Einstein's identification of the speed of light as a term instrumental in describing the curvature of space. The quantized expressions presented here are notably independent of the fundamental units of measure and as such reveal that macroscopic terms (i.e., c, v), inclusive of measure, introduce unnecessary descriptors into our understanding of nature. Rather, contraction and dilation are a function of counts of invariant fundamental measures, specifically $n_{L o}, n_{L l}$ and $n_{M}$ with respect to a center of gravity $n_{L r}$. We may make two observations.

$O_{4}$ : The measurement distortion with respect to motion includes a squared parameter $n_{L m}^{2} / n_{L c}^{2}$. Conversely, measurement distortion with respect to gravitation is a first-order phenomenon $n_{M} / n_{L r}$

$O_{5}$ : The measurement distortion with respect to gravitation is "not" a stretching of $I_{f}$ (i.e., of space), but a count differential.

Notably, where Informativity approaches space-time curvature as a limitation of physically significant bounds to measure, Loop Quantum Gravity (LQG) has played a long role in the description of a quantized space from a different point of view. [6] That is, LQG uses a discrete geometry which first arose from solutions to the spectral problem. Of particular interest is the convergence between the canonical quantization of GR and the same where resolved from the covariant Ooguri theory to reduce the background field (BF) to gravity. Collectively these successes provide a framework connecting Einstein's GR expressions with the Informativity space-time model, the later which operates at a fundamental scale.

\subsection{Frames of Reference}

Up to this point, our focus has been on how a measure may only be understood with respect to the remaining two measures. As such, we have described some expressions as self-referencing-but what does this mean?

When anchored by the fundamental expression $l_{f} m_{f}=2 \theta_{s i} t_{f}$ each of the three measures may be understood only in the context of the remaining two. That is, we may understand the fundamental measures with no other measure or logical relation that adds additional meaning to any of the measures. This is why we refer to fundamental measures collectively as self-referencing.

A framework is defined as a set of three references, each assigned to one of the three measures. Examples include the International System of Units (SI), Planck's Units, and the fundamental units. Regardless of which set is used, a translation between frameworks is always possible. Thus, frameworks do not change our descriptions of phenomena, but some frameworks can provide more detail and assist in recognizing relationships that are physically significant in nature.

There are phenomena that are better described with a framework defined with 
respect to the universe. Such a framework would include counts of the fundamental measures where the count $n_{T u}$ of $t_{f}$ is defined with respect to the time elapsed since the dawn of the universe. Expressions using terms related to this framework are called self-defining in part because the universe does not have an external reference with which to compare a measure. The self-defining measures are the diameter $D_{U}$, mass $M_{U}$, and age $A_{U}$ of the universe. Likewise, the corresponding self-defining counts of the fundamental measures are $n_{L u}, n_{M u}$ and $n_{T u}$ Because each of the measures represents an upper count bound to the phenomenon being described, the terms are additionally distinguished as system parameters.

A mathematical approach to resolving values for system parameters is to first take the fundamental expression, set a fundamental measure to a value of one, and then resolve the corresponding count ratio for the remaining two. Applying this approach to mass, then

$$
\begin{gathered}
m_{u}=\frac{2 \theta_{s i}}{c} \frac{n_{T u}}{n_{L u}}=1, \\
\frac{2 \theta_{s i}}{c}=\frac{n_{L u}}{n_{T u}}, \\
m_{f}=\frac{n_{L u}}{n_{T u}} .
\end{gathered}
$$

Here $n_{L u}$ is a count of $l_{f}$ and $n_{T u}$ is a count of $t_{f}$ that describe the universe. We may now describe phenomena which are self-defining. For example, several expressions with the same precision and value as our best measurement data concern mass accretion $M_{\text {acr }}$ (a count of $m_{f}$ per count of $t_{f}$ ), the diameter $D_{U}$ and age $A_{U}$ of the universe, and the expansion of the universe $H_{U}$ ([1] Equations (135), (89), (90)), respectively

$$
\begin{gathered}
M_{a c r}=\frac{n_{M u}}{n_{T u}}=\theta_{s i}\left(\frac{\theta_{s i}^{2}+2}{2}-1\right)=\frac{\theta_{s i}^{3}}{2}, \\
D_{U}=\frac{n_{L u}}{n_{T u}} c A_{U}, \\
H_{U}=\frac{n_{L u}}{n_{T u}} c=2 \theta_{s i} .
\end{gathered}
$$

Understanding self-referencing and self-defining frameworks is also important in balancing units in Informativity expressions. Whereas the units for certain terms may differ in different frames of reference, the values associated with those terms are the same. This quality is central to understanding how nondimensionalization applies to measurement quantization, especially the more complex expressions such as angular measure $\theta_{s i}=h / 4 \pi l_{f}$ and momentum $\theta_{s i}=$ $I_{\mathcal{F}} \hat{c}^{3} / 2 G$, which if set equal to one another resolve the well-known expression for Planck's length, [14]

$$
l_{p}=\left(\frac{\hbar G}{c^{3}}\right)^{1 / 2} \mathrm{~m} .
$$


Unit analysis will not be central to the discussions within this paper. Nevertheless, the change in nomenclature from fundamental length $I_{f}$ to Planck's length $l_{p}$ is physically significant and the two are related.

\subsection{Mass Distribution as a Property of the Universe}

The effects of measurement distortion will appear anywhere distance is considered. We emphasize that the construction of expressions with respect to the universe as a framework can more readily reveal relations that are not so nicely resolved when the frame of reference chosen is the local inertial frame.

Framework consideration is important when describing events that occurred during the early formation of the universe and when describing phenomena that are best described as system properties where the universe is the system.

By example, consider the expansion. In the local frame, universal expansion varies with time as expressed by $H=\left(\mathrm{km} \cdot \mathrm{Mpc}^{-1}\right) / A_{U}=70.860 \mathrm{~km} \cdot \mathrm{s}^{-1} \cdot \mathrm{Mpc}^{-1}$. With time in the denominator, this expansion expression is decreasing but when expressed with respect to the universe, the expansion is invariant $H_{U}=2 \theta_{s i} \mathrm{ly} \cdot \mathrm{y}^{-1}$. Notably, the former indicates only a rate of expansion and tells us little more. The invariant expression though tells us that expansion is an intrinsic property of the universe. Indeed, it is fixed and its invariance provides insight into a law of nature that is fundamental to our universe. Note lastly that the expression describes universal expansion, not stellar expansion.

Without this deeper understanding of the relationship between our inertial frame and the universe, it is difficult to apply the effects of measurement distortion to phenomena that are cosmological in scale.

In a further example, let us explore how viewing the universe as a frame of reference can have a significant effect on our understanding of mass. We begin by citing the mass distribution expression ([1], Equation (118)) where

$$
2 M_{t o t} M_{f}=M_{o b s}\left(M_{t o t}+M_{f}\right) .
$$

The expression describes the relationship between total $M_{\text {tot }}$ and observable $M_{o b s}$ mass in the universe. Fundamental mass $M_{f}$ ([1], Equation (93)) is new to modern theory and will require a short introduction.

The principles that constrain fundamental mass are as follows. All measure is subject to an upper frequency of $1.85492 \times 10^{43}$ events per second, Equation (38), and we call that upper bound the mass frequency. If a greater number of mass events should reach an observer, there would be no means to distinguish them. Mass frequency defines an upper bound to the total mass that may be observed. We recognize this bound with the term $M_{f}$ which has the formal definition

$$
M_{f}=A_{U} \theta_{s i} \frac{m_{f}}{t_{f}}=5.7353 \times 10^{53} \mathrm{~kg}
$$

With this, we may now place the mass distribution expression into Pythagorean form. First, we divide Equation (89) by $m_{f}^{2}$. Thus, as presented in Figure 4 , where the total $n_{\text {Mtot }}$ observable $n_{\text {Mobs }}$ and fundamental $n_{M f}$ counts of $m_{f}$ for 


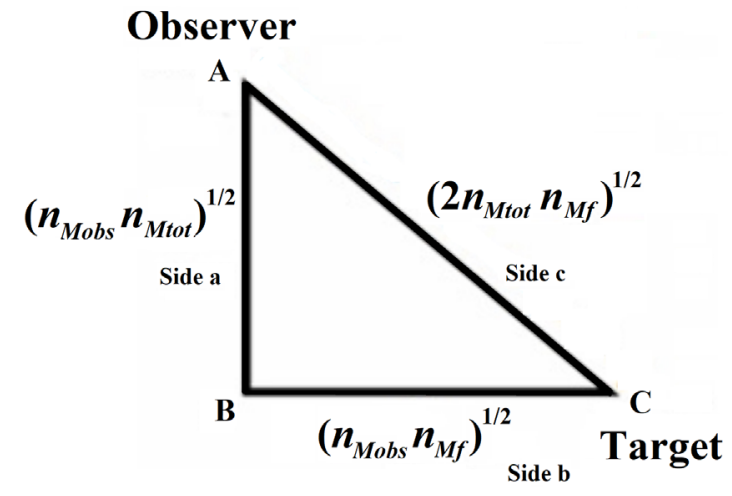

Figure 4. Mass distributions of the universe.

the universe are compared with the expression for length contraction

$$
\begin{gathered}
n_{L o}^{2} n_{L c}^{2}+n_{L m}^{2} n_{L l}^{2}=n_{L c}^{2} n_{L l}^{2}, \\
n_{M o b s} n_{M t o t}+n_{M o b s} n_{M f}=2 n_{M t o t} n_{M f} .
\end{gathered}
$$

we find a one-to-one correspondence for each term:

- $n_{L o}^{2} \triangleq n_{\text {Mobs }}$ : observed;

- $n_{L m}^{2} \hat{=} n_{\text {Mobs }}$ : change between frames;

- $n_{L l}^{2} \hat{=} n_{M f}:$ local;

- $n_{L c}^{2} \hat{=} n_{\text {Mtot }}$ : upper bound.

The two expressions are correlated with length contraction being a composite expression consisting of counts of $l_{f}$ defined with respect to the local frame (self-referencing), and mass distribution being a composite expression of counts of $m_{f}$ defined with respect to the universe (self-defining).

The general form for the mass distribution, Equation (89), is more versatile as the terms may take mass values in kilograms or percentage distributions with respect to the total. Then, finally, when both expressions are in the same form, we may now present the mass distribution as a relativistic expression,

$$
M_{o b s}=M_{f}\left(2-\frac{M_{o b s}}{M_{t o t}}\right)
$$

describing the observed mass $M_{o b s}$ as the fundamental mass $M_{f}$ after applying the effects of measurement distortion. Note that $M_{o b s} / M_{t o t}$ is a first-order gravitational parameter and does not take the squared form of the speed parameter $\left(n_{L m}^{2} / n_{L c}^{2}\right)$. Likewise, comparing gravitational motion with the definition of the mass distribution, the observable mass $M_{o b s}$ is written with respect to the upper bound $M_{\text {tot }}$

The relativistic correlation is significant in that it validates our understanding of fundamental mass $M_{f}$ as an upper bound to the observation of mass events. Second, the expression provides a conversion metric between measures in the local frame with respect to the self-defining frame (i.e., defined with respect to the universe). Understanding frames of reference is important when resolving conditions prior and up to the trigger event that initiates expansion. For brevity, 
we symbolically identify this period as the quantum inflationary epoch although distinctly different from $t_{0}$.

\subsection{Cosmic Microwave Background}

With Informativity, we can present expressions that describe the dawn of the universe from a quantum fluctuation, the ensuing inflationary expansion and the trigger event (i.e., $R_{U}=\sqrt{3} l_{f}$ ) that causes inflation to cease, and no longer constrains the accumulating mass/energy

$$
M_{a c r}=\frac{\theta_{s i}^{3} m_{f}}{2 t_{f}}=\frac{\theta_{s i}^{4}}{l_{f}}=7.00888 \times 10^{36} \mathrm{~kg} / \mathrm{s}
$$

at $A_{U}=\mathrm{e}^{\sqrt{3} \theta_{s i}^{3} / 2}$ seconds as Cosmic Microwave Background (CMB) ([1] Sections 3.13 - 3.15). Here the focus is not on a comprehensive review of those calculations. Rather, we explore why the calculated age of the CMB points to an elapsed time of 363,309 years [15] whereas the events that end inflation define a trigger at 678,889 years.

Time dilation is the factor at play. We must account for the measurement distortion between the quantum events that led to the conclusion of CMB production and our inertial frame within a universe expanding at $H_{U}=2 \theta_{s i}$ Taking the integral of the radius $R_{U}=\sqrt{3}$ as described during quantum inflation at the time of the CMB trigger event, we may solve for the age of the universe ([1], Equations (151) and (152)),

$$
\begin{gathered}
R_{U}=\int \frac{2 l_{f}}{\theta_{s i}^{3} A_{U}}=\frac{2 \ln \left(A_{U}\right) l_{f}}{\theta_{s i}^{3}}, \\
A_{U}=\mathrm{e}^{\sqrt{3} \theta_{s i}^{3} / 2}=1.14652 \times 10^{13} \mathrm{~s} .
\end{gathered}
$$

Using the "expansion" expression for the radius of the universe $R_{U}=A_{U} \theta_{s i} c$ from Equation (86), then the difference between the self-referencing age $A_{s-\text { ref }}$ and the self-defining age $A_{U}$ is a function of volume where $V_{U}=(4 / 3) \pi R^{3}$ such that

$$
\begin{gathered}
\frac{(4 / 3) \pi\left(A_{s-r e f} \theta_{s i} c\right)^{3}}{(4 / 3) \pi\left(A_{U} \theta_{s i} c\right)^{3}}=2 \theta_{s i}, \\
A_{s-\text { ref }}=\left(2 \theta_{s i}\right)^{1 / 3} A_{U}=2.14241 \times 10^{13} \mathrm{~s} .
\end{gathered}
$$

To resolve the time dilation between the two frames of reference, we organize time from Equation (33) with respect to motion such that

$$
\frac{n_{T o}}{n_{T l}}=\left(1-\frac{n_{L m}^{2}}{n_{L c}^{2}}\right)^{1 / 2} .
$$

Then we modify the expression for the self-referencing age, where $A_{s-\text { ref }}=n_{T A} l_{f}$ is given in the local frame during quantum inflation, and $A_{U}=n_{T o} l_{f}$ is given in the observed frame as viewed from our perspective today, to get 


$$
\begin{gathered}
A_{s-r e f}=A_{U}\left(2 \theta_{s i}\right)^{1 / 3}, \\
n_{T l}=n_{T o}\left(2 \theta_{s i}\right)^{1 / 3}, \\
\frac{n_{T o}}{n_{T l}}=\frac{1}{\left(2 \theta_{s i}\right)^{1 / 3}} .
\end{gathered}
$$

Setting the expressions equal to one another, we then resolve the length contraction between the two frames,

$$
\begin{gathered}
\frac{1}{\left(2 \theta_{s i}\right)^{1 / 3}}=\left(1-\frac{n_{L m}^{2}}{n_{L c}^{2}}\right)^{1 / 2}, \\
n_{L m}^{2}=n_{L c}^{2}-\frac{n_{L c}^{2}}{\left(2 \theta_{s i}\right)^{2 / 3}}, \\
n_{L m}=n_{L c} \sqrt{1-\frac{1}{\left(2 \theta_{s i}\right)^{2 / 3}}} .
\end{gathered}
$$

Note, this is a second-order contraction expression and as such does not need the inside-out transform we performed for mass in Equations (72) and (73). As expected, we find that the speed parameter $1^{2} /\left(\left(2 \theta_{s i}\right)^{1 / 3}\right)^{2}$ corresponds to Einstein's speed parameter $v^{2} / \mathrm{c}^{2}$, but the frame of reference is the universe. We cannot set the parameters equal in that doing so mixes two frames of reference, the local inertial frame with that of the universe.

Multiplying Equation (104) by $l_{f} / n_{T} t_{f}$ we resolve a contraction effect that corresponds to a velocity of

$$
\frac{n_{L m}}{n_{L c}}=\frac{n_{L m} l_{f}}{n_{T} t_{f}} \frac{n_{T} t_{f}}{n_{L c} l_{f}}=\frac{v}{c}=\sqrt{1-\frac{1}{\left(2 \theta_{s i}\right)^{2 / 3}}}=84.4755 \%
$$

of the speed of light. Note that $n_{T}=\left(1 / t_{t}\right)$ is fixed by our definition of $n_{L c}$ in SI units. The terms cancel multiplicatively, but are retained for consistency in structure.

Again, we are not just resolving the associated measurement distortion between two frames of reference. The magnitude of the time dilation is fixed by the expansion parameter $2 \theta_{s i}$, which describes the self-referencing lower bound with respect to the three-dimensional volume of radius $\sqrt{3} l_{f}$. The trigger event $\sqrt{3}>1 / 2>\sqrt{2}$ where $\sqrt{2}$ rounds down and

$$
\begin{gathered}
\sqrt{a^{2}+b^{2}}=\sqrt{a^{2}+{\sqrt{a^{2}+b^{2}}}^{2}} \\
\sqrt{a^{2}+a^{2}+b^{2}}=\sqrt{1^{2}+1^{2}+1^{2}}=\sqrt{3}
\end{gathered}
$$

rounds up is what determines the precise elapsed time and associated space within the scope of the spatial framework.

As a technical note, side $\mathbf{b}$ is not arbitrary. It is the only relation that can be resolved with the reference, the hypotenuse of a Pythagorean triangle with sides $\mathbf{a}=1$ and $\mathbf{b}=1$. Therefore, the square root of $a^{2}+b^{2}$ becomes the square root of $a^{2}+\left(\left(a^{2}+b^{2}\right)^{1 / 2}\right)^{2}$. 
With a system radius of $\sqrt{3} l_{f}$, points outside of the bubble come into view, quantum inflation ends and the measurement framework expands at the speed of light.

Given that the focus is on measurement distortion, a better explanation of the transition from quantum inflation to expansion has been a subject of interest. This can be explained without taking a significant tangent.

Imagine the universe today. There are many points in space we might choose to reach, but there are constraints. For one, the rate of expansion which describes length change in the referential framework (i.e., the set of points an inertial frame can reach) may not exceed the speed of light with respect to points at the outer edge of that framework. We also recognize that the rate of expansion is attained only when there are points that can be plotted as described by the Pythagorean Theorem with respect to a whole-unit count of the reference $I_{f}$

Consider then, a universe that has a radius of $\sqrt{2} l_{f}$. How do you get past a point that is $1.414 l_{f}$ in distance with your reference measure $I_{f}$ (which must be a whole-unit count of $l_{f}$ ) and your definition of distance $a^{2}+b^{2}=c^{2}$ where $a, b$, and $c$ are all whole-unit counts of $l_{f}$. Indeed, you cannot. The space has a radius of $l_{\dot{p}}$ that being $\sqrt{2} l_{f}$ rounded down to $I_{f}$ the closest whole-unit count of the reference. In that the reference describes the smallest physically significant distance, there exists only one reference point where distance has significance.

Nonetheless, this does not imply that the system is static. Bounds to a measure do not imply that the properties of the system are necessarily constrained in the same way (see $O_{1}$ and $O_{3}$ ). With regards to this quantum bubble, the calculated radius is a function of time and may be resolved where $R_{U}=2 \ln \left(A_{U}\right) l_{f} / \theta_{s i}^{3}$ from Equation (95).

The radius increases at a quantum rate for 363,309 years, Equation (96), until it reaches $\sqrt{3} l_{f}=1.732 l_{f}$. This value will round up to a whole-unit count of two, which means that new particles may now appear outside of the quantum bubble. The volume of the universe immediately proceeds to expand at the speed of light to a radius of $\sqrt{4} l_{f}$ without problem. The expansion continues to a radius of $\sqrt{5} l_{f}=2.236 l_{f}$, which rounds down. Why does the expansion not stop?

Expansion does not stop because with a larger universe we now have new points of reference inside the universe. Some of those points are $\sqrt{3} l_{f}$ from the outer edge of the quantum bubble. With our larger universe, there are always points that are $\sqrt{3}_{f}$ from the outer edge. Expansion is now the new behavior and from Equations (86) and (87) the radius obeys

$$
R_{U}=A_{U} \theta_{s i} \frac{l_{f}}{t_{f}}
$$

where $c=l_{f} / t_{f}$ is given in SI units.

If it were not for quantum inflation, mass/energy accretion, and an upper bound to mass density $2 n_{M} / n_{L r}$, conditions would not exist that lead to the addition of energy in the form of electromagnetic radiation. During the quantum inflationary epoch, the addition of energy exceeded the upper bound to mass 
density as described in Equation (79). Energy accretion was restricted to non-baryonic forms and it is these conditions that constrained the universe to a very specific quantity, age, density, and temperature of the $\mathrm{CMB}$, as described in the first paper [1].

\subsection{What Defines Measure?}

Why is the fundamental measure for length $I_{f}=2 G \theta_{s i} / c^{3}=1.61620 \times 10^{-35} \mathrm{~m}$ ? We know its relation to the other measures as given by the fundamental expression $l_{f} m_{f}=2 \theta_{s i} t_{f}$ Therefore, what property of our universe anchors its value? Does it vary and, if not, then why is it invariant?

We begin our analysis by first recognizing that progress can come only by looking to disciplines outside of measure. In this section, we entertain the idea that counts of the fundamental measures guide our understanding of their relationships and constrain their values. Taking Equation (103) and arranging it in the form $a^{2}+b^{2}=c^{2}$, then

$$
\frac{1}{\left(2 \theta_{s i}\right)^{2 / 3}}+\frac{n_{L m}^{2}}{n_{L c}^{2}}=1 .
$$

The two components are anchored by the value 1 . There is a minimum, a component which represents the lower count bound to measure $1 /\left(2 \theta_{s i}\right)^{2 / 3}$. There is also a maximum, a component which represents the upper count bound to measure $n_{L m}^{2} / n_{L c}^{2}$. Note that $n_{L m}$ is a fixed value that corresponds to expansion.

Next, we need to separate the squared terms from their components. We also want to replace $2 \theta_{s i}$ with the corresponding fundamental measures that we seek to define. Moreover, we want to correlate the physical description depicted in Figure 5. We call this a unity expression,

$$
\left(\left(\frac{t_{f}}{l_{f} m_{f}}\right)^{1 / 3}\right)^{2}+\left(\frac{n_{L m}}{n_{L c}}\right)^{2}=1 .
$$

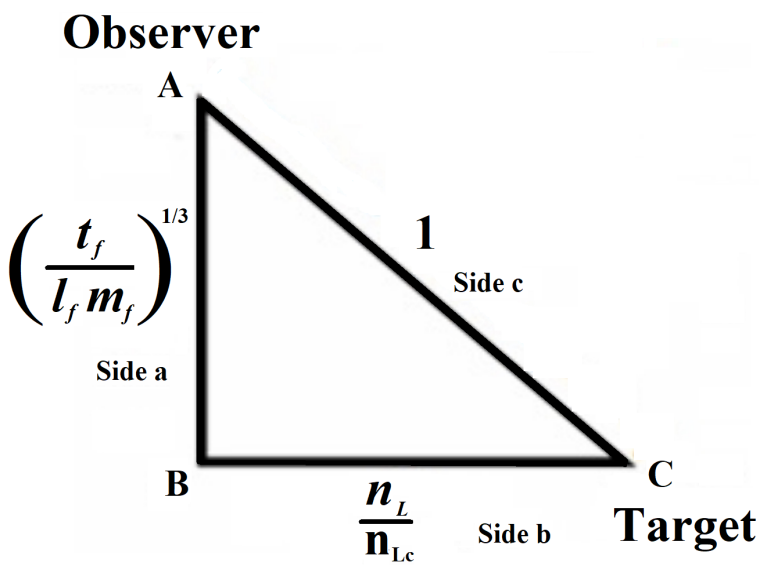

Figure 5. The unity expression, the lower (side a) and upper bound (side b) metrics that define unity. 
Having used the fundamental expression, the first term now contains each of the three measures, and for each dimension, we must take the root to conform to the term in the one-dimensional component term (side a) of the Pythagorean Theorem. Second, the three measures are the lower bound terms that define length, mass and time; the product of length and mass $\left(3.51755 \times 10^{-43} \mathrm{~kg} \cdot \mathrm{m}\right)$ being the smallest significant value with respect to time $\left(5.39106 \times 10^{-44} \mathrm{~s}\right)$, the smallest value.

The second component is a count relation representing the upper bound portion of the relation. It consists of a ratio that describes the motion associated with expansion $n_{L m}$ with respect to the upper bound $n_{L c}$ Together, the expression correlates measure with counts of those measures.

Lastly, whereas the units in the second term $n_{L m} / n_{L c}$ cancel, the leftmost term has units $\mathrm{s} / \mathrm{kg} \mathrm{m}$ which combine to provide unity. This is not to say that they should or should not cancel. We can only infer that:

$O_{6}:$ Our study of both the unity and fundamental expressions continues to reinforce the idea of equality between time and the product of length and mass.

When we apply this principle, using a maximum or minimum bound as our frame of reference, we find that $\theta_{s i}$ carries no units at all. Certainly, it may be argued that when focusing solely on the fundamental expression that $\theta_{s i}$ carries the inverse units $\mathrm{kg} \mathrm{m} / \mathrm{s}$, but we are regularly reminded that this assignment meets with conflict as demonstrated above. Changes in the reference framework change the units under consideration.

If we were now to consider a universe that differs from our own, then that difference would be expressed here. Any change in the rate of expansion $H_{U}=$ $2 \theta_{s i}=n_{L u} c / n_{T u}([1]$, Equation (81)) identifies only one set of fundamental measures in regard to that of the universe. The self-defining expression (i.e., defined with respect to the universe) for mass is ([1], Equation (79))

$$
m_{f}=\frac{n_{L u}}{n_{T u} c},
$$

where $n_{L u}$ is the diameter per light-year and $n_{T u}$ is the age per year. Multiplication of $n_{T u}$ by $\mathrm{c}$ is necessary for SI units. Note that the remaining two measures $l_{f}$ $=n_{M u} / n_{T u}$ and $t_{f}=1 / n_{M u} n_{L u}\left([1]\right.$, Appendix D) are superfluous because $n_{M u} \sim^{\sim} n_{T u}$, unlike mass where $n_{L u} \propto n_{T u}$.

The self-referencing expressions (i.e., defined with respect to the local frame) are

$$
\begin{gathered}
l_{f}=\frac{2 G \theta_{s i}}{c^{3}}, \\
t_{f}=\frac{2 G \theta_{s i}}{c^{4}}, \\
m_{f}=2 \frac{\theta_{s i}}{c} .
\end{gathered}
$$


They may not be factored of fundamental measures but may incorporate measure into an invariant bound such as $\theta_{s i}, G$ or $c$. We may even expand a measure to match an upper system bound such as the diameter $D_{U}$ in light-years and age $A_{U}$ in years of the universe,

$$
m_{f}=\frac{D_{U}}{A_{U} c} .
$$

With $H_{U}=2 \theta_{s i}$ and $m_{f}=2 \theta_{s i} / c$, we may combine the self-defining system constant $H_{U}$ with the self-referencing measures to show their correlation,

$$
H_{U}=\frac{m_{f} l_{f}}{t_{f}} .
$$

As such,

$$
\left(\left(\frac{1}{H_{U}}\right)^{1 / 3}\right)^{2}+\left(\frac{n_{L m}}{n_{L c}}\right)^{2}=1 .
$$

Sides $\mathbf{a}$ and $\mathbf{b}$ of this Pythagorean expression are an invariant description of $\mathbf{a}$ three-dimensional space-time expansion. $\left(1 / H_{U}\right)^{1 / 3}$ describes 'three' dimensions and their correspondence to length $n_{L m} / n_{L c}$. That is, the measure of length is fixed by the equality. Each measure is also anchored as described by the fundamental expression.

$\mathrm{O}_{7}$ : While mathematical manipulation of physical description can offer us a many dimensional universe, an Informativity description of measure consistently offers only three dimensions.

We may also attach importance to measure as a tangible object of physical significance. We can, for instance, demonstrate that their relation identifies a fixed set of parameters that describe invariant properties of the universe such as mass $n_{M u}$ diameter $n_{L u}$ age $n_{T u}$ and expansion $l_{f} m_{f} / t_{f}$

$O_{8}$ : One might ask if system parameters are a required numerical outcome, then do the phenomena they describe have significance beyond their mathematical certainty?

Lastly, using Equation (112) to describe c, we find that

$$
\begin{gathered}
c=\frac{n_{L u}}{n_{T u} m_{f}} \\
l_{f} m_{f}=\frac{n_{L u}}{n_{T u}} t_{f}
\end{gathered}
$$

which gives us the fundamental expression where the frame of reference is the universe. However, if $c=n_{L} l_{f} / n_{T} t_{f}$ in the local frame such that $n_{L}=n_{T}$, then applied to the self-defining frame we get

$$
c=\frac{n_{L u}}{n_{T u}} \frac{1}{m_{f}}=\frac{1}{m_{f}}
$$


which is false. The paradox clarifies that length, mass, and time are not conserved between the local and universal frames of reference. The analysis in the first paper demonstrates that mass accretes at a steady rate of ([1], Equation (136))

$$
M_{a c r}=\frac{n_{M u}}{n_{T u}} \frac{m_{f}}{t_{f}}=\frac{\theta_{s i}^{3} m_{f}}{2 t_{f}}=7.00888 \times 10^{36} \mathrm{~kg} / \mathrm{s} .
$$

$O_{9}$ : Where length, mass, and time do not correspond between the local frame of reference and that of the universe, we find that the mass of the universe increases with elapsed time.

Notably, the expression may also be written as $M_{a c r}=\theta_{s i}^{4} / l_{f}$. If mass accretion was not a property of the universe, not only would there be a conflict with our understanding of the speed of light, but we would be unable to resolve the age, quantity, density and temperature of the CMB. Those calculations match observational data to four significant digits and are predicated on mass accumulating with elapsed time.

\subsection{The Quantum Crossover}

An understanding of measure should not occur without also bringing to your attention that the availability of information does not only affect measure. The availability of information also defines the quantum crossover-the attributes of a system which separate classical and quantum physics into two distinct classes of behavior. Until now, where this division occurs and why it exists has been elusive. Recall, Equation (1) defines one aspect of this division with respect to quantum uncertainty as $n_{M} n_{L r} n_{L} \geq I_{f}$ The crossover is not entirely clear for all systems especially where quantum behavior can occur with respect to macroscopic phenomena.

$O_{10}$ : Those qualities of a system that divide the classical and quantum behavior of matter into two classes are a function of what information is available to the observer. That information regards the existence or lack thereof of an event.

By example, consider the position and momentum of an electron. The position of the electron has a precise location at all times, but it is not known nor can it be known as precisely in combination with the momentum. The quantum description provided by the wave function is the most appropriate in part because we seek to know the position of an object that is itself less in mass than the reference $m_{f}$

But, this uncertainty in position should not be confused with the "uncertainty" that prevents the observer from precisely measuring position and momentum. What information is available is a distinctly separate behavior that arises from upper and lower bounds to measurement frequency particular to the system.

The wave function $\Psi$ describes positional uncertainty, but what is going on underneath is less defined. That said, describing the accessibility of information as a collapse of the wave function can be less defining. The example entertains 
only the presence or absence of information available to the observer. The importance of information has always been evident in modern theory. What has not been evident is a model of quantum physics that formally separates where the classical laws of physical behavior cease and where the quantum laws begin. Informativity provides that model, Equation (4) being one example of its application.

From a more philosophical perspective, Einstein summarized his interpretation of objective reality when he asked Abraham Pais "whether [he] really believed that the moon exists only when I look at it" [16]. In light of Informativity, Einstein's concern is protected; an observer does not need to receive information in order for a mass to exist or an event to occur. But what the observer's behavior will be will follow as though the mass or event did not exist and/or never occurred until that information reaches the observer.

We should take this moment to also note where Heisenberg's Uncertainty Principle resolves the crossover for fundamental length, substituting $t_{f}$ for $l_{f}$ in Equation (4) using the fundamental expression and Equation (87), then the corresponding crossover for time is

$$
\begin{gathered}
l_{f}=2 \theta_{s i} \frac{t_{f}}{m_{f}}=\frac{n_{L u}}{n_{T u}} \frac{t_{f}}{m_{f}}, \\
\frac{n_{M} n_{L r} n_{L} n_{T u} m_{f}}{n_{L u}} \geq t_{f} .
\end{gathered}
$$

Something more approachable would be to take Heisenberg's Uncertainty Principle with respect to the position and momentum of a particle $\sigma_{X} \sigma_{P} \geq \hbar / 2$ where $\hbar / 2=\theta_{s i} l_{f}\left([1]\right.$, Equation (35)) and where universal expansion is $H_{U}=2 \theta_{s i}$ and write

$$
\begin{gathered}
\sigma_{X} \sigma_{P} \geq \theta_{s i} l_{f}, \\
2 \sigma_{X} \sigma_{P} \geq H_{U} l_{f} .
\end{gathered}
$$

Then the uncertainty in position and momentum describe a threshold where the radial expansion of the universe $H_{U} / 2$ multiplied by $l_{f}$ defines the threshold.

\subsection{Relation and Boundary Expressions}

We conclude our discussion on measurement distortion by emphasizing two classes of expressions that are physically distinct: relations and bounds.

The conjecture is that all relations may be reduced to the fundamental expression, $l_{f} m_{f}=2 \theta_{s i} t_{f}$ Relations such as the distribution of mass-visible, observable, fundamental, dark and total-are modifications of the fundamental expression. Other examples include the diameter $D_{U}$ and age $A_{U}$ of the universe as well as the constants associated with Planck $\hbar$ and Newton $G$ ([1], Equations (118), (87) and (40)),

$$
M_{o b s} M_{t o t}+M_{o b s} M_{f}=2 M_{t o t} M_{f}
$$




$$
\begin{gathered}
D_{U}=2 \theta_{s i} A_{U}, \\
4 G \theta_{s i}^{2}=\hbar c^{3} .
\end{gathered}
$$

However, not all principles, laws or rules of nature are relations. There are also bounds, such as the speed of light $c$, maximum mass density $2 n_{M} / n_{L r}$, and the gravitational constant $G$, respectively,

$$
\begin{gathered}
c=l_{f} / t_{f}, \\
2 \frac{n_{M}}{n_{L r}}<1, \\
G=\frac{l_{f}}{t_{f}} \frac{l_{f}}{t_{f}} \frac{l_{f}}{t_{f}} \frac{t_{f}}{m_{f}} .
\end{gathered}
$$

These expressions are important because they define upper and lower bounds to length, mass, time frequency, and combinations thereof. Bounds may incorporate relations and may also contain variables that are specific to the scope of measured phenomena. We may, for instance, use a relation such as the fundamental expression, to make similar substitutions to a bound and resolve other bounds. Starting with the expression for the gravitational constant $G$, we may resolve the associated velocity,

$$
\begin{gathered}
\frac{G m_{f}}{l_{f}}=\frac{l_{f}}{t_{f}} \frac{l_{f}}{t_{f}}, \\
\sqrt{2} c=\left(\frac{2 G m_{f}}{l_{f}}\right)^{1 / 2}, \\
\sqrt{1^{2}+1^{2}} c=\left(\frac{2 G m_{f}}{l_{f}}\right)^{1 / 2} .
\end{gathered}
$$

The bound for velocity $v=(2 G M / r)^{1 / 2}$ arises from the expression for gravity. When multiplied by $t_{f}$

$$
\sqrt{1^{2}+1^{2}} l_{f}=t_{f}\left(\frac{2 G m_{f}}{l_{f}}\right)^{1 / 2}
$$

the expression describes distance using the Pythagorean Theorem. Naturally, as $c=l_{f} / t_{f}$ the lower bound to velocity times $t_{f}$ is $\sqrt{2}$ units of $l_{f}$ which resolves the reference $l_{f}$ on side a defined against itself, $l_{f}$ on side $\mathbf{b}$. Finally, we generalize the expression as performed in Equations (59)-(64).

Distinguishing relations from bounds is instrumental in recognizing that the development of a "unified field equation" implies that each of the four forces is a measurement bound. While this principle has been demonstrated for gravity, the construct for each of the three remaining forces is still a task for future research.

\subsection{Deflection of Light near the Sun}

Experimentally there are several approaches that may be taken to validate mea- 
surement quantization. That is, the predictions of Informativity do add some quantum detail where GR drops off. For one, there is an entirely new effect of measure distortion described by Informativity, which we have termed the Informativity differential. We do not present all possible experiments, as those proposals may be found in the first paper [1], but we discuss this new effect regarding the deflection of light grazing the Sun.

This effect is six orders in magnitude smaller than that described by GR, $\theta=$ $4 G M / r C^{2}=8.5 \times 10^{-6} \mathrm{rad}$. Using the same expression, we may resolve the difference between GR and the Informativity differential by replacing Newton's gravitational term $G / r^{2}$ as a difference $Q_{L} c^{3} / r \theta_{s i}$ thus revealing that the additional curvature due to the Informativity differential is

$$
\Delta \theta=\left(\frac{G}{r^{2}}-\frac{Q_{L} c^{3}}{r \theta_{s i}}\right) \frac{4 r M}{c^{2}}=\frac{4 M\left(G \theta_{s i}-Q_{L} r c^{3}\right)}{r c^{2} \theta_{s i}},
$$

$$
\Delta \theta=\frac{4 \times 1.98855 \times 10^{30}\left(\left(6.67408 \times 10^{-11} \times 3.26239\right)-\left(1.15956306 \times 10^{-44} \times 6.969 \times 10^{8} \times 299792458^{3}\right)\right)}{6.969 \times 10^{8} \times 299792458^{2} \times 3.26239}
$$

$$
\Delta \theta=6.6 \times 10^{-12} \mathrm{rad} .
$$

The calculation is sensitive to the number of digits used in $Q_{L}$ which is not a measured value.

The experiment would provide a new approach to confirming the physical significance of the fundamental units and their application to gravitational fields. While several experiments described in the first paper are already well tested and do conform to this model, experiments that expose these quantum effects in a gravitational field would provide a new perspective.

\section{Discussion}

Measurement quantization has revealed several notable properties that apply to our understanding of space-time, to our understanding of relativity, and our understanding of cosmological phenomena, and hence are presented as properties of our universe. While we may describe these phenomena in several mathematical forms, if it were not for measurement quantization, we would not see the numerical qualities of measurement distortion and how the Pythagorean Theorem plays into that description. We would also have no recourse with which to gain a greater understanding of measure, constrained to a self-referencing framework of fundamental measures.

Informativity brings us not just a new model of physical expression but focuses our attention on the self-defining framework as a means of understanding cosmological phenomena. With measurement quantization, we are able to provide a more refined understanding of gravitation, of the relations that underlie equivalence, and as expressed in the unity expression, an underlying description of measure and the geometric constraints that bind their values.

Notably, we are able to expand on our understanding of measure to include 
not only the self-referencing inertial frame of the observer, but also the self-defining frame of the universe. Without this broader understanding, we would find ourselves without a firm understanding of some of the greater cosmological mysteries such as dark energy, dark matter, dark mass, and the expansion of the universe.

\section{Glossary of Terms}

\section{Boundary Expressions}

Expressions define invariant upper and lower bounds to length, mass, time frequency, and combinations thereof. Examples include $c=l_{f} / t_{f} G=\left(l_{f} / t_{f}\right)^{3}\left(t_{f} / m_{f}\right)$, $\hbar=2 \theta_{s i} l_{f}$ and $H_{U}=l_{f} m_{f} t_{f}$

\section{Framework}

A frame of reference against which a system of measure is applied. Frameworks are commonly discussed in Informativity and are typically either that of the observer's inertial frame, the observed target or that of the universe.

\section{Fundamental Expression}

The simplest expression correlates the three fundamental measures, $l_{f} m_{f}=$ $2 \theta_{s i} t_{f}$

\section{Fundamental Measure}

One of the measures length $l_{f}$ mass $m_{f}$ and time $t_{f}$ along with their correlation called the fundamental expression. Using measurement data from the Shwartz and Harris experiments in combination with Heisenberg's Uncertainty Principle, each is macroscopically defined and physically significant.

\section{Informativity Differential}

The Informativity differential $Q_{L} n_{L r}$ describes a new form of length contraction associated with the lower bound to measure. The loss of immeasurable space at each increment of $t_{f}$ describes gravity.

\section{Measurement Distortion}

A short-hand notation for the contraction and dilation of measure.

\section{Relation Expressions}

Any expression that may be reduced to the fundamental expression, $l_{f} m_{f}=$ $2 \theta_{s i} t_{f}$ Examples include universal mass distribution and the correlation of the diameter to the age of the universe.

\section{Self-referencing}

An expression defined with respect to the observer's inertial frame of reference.

\section{Self-defining}

An expression defined with respect to the universe as a frame of reference.

\section{System Parameters}

Any invariant value associated with a self-defining expression.

\section{Unity Expression}

A self-defining Pythagorean expression with terms describing measurement bounds and a hypotenuse equals to 1 . 


\section{Acknowledgements}

We thank Edanz Group

(https://www.edanzediting.com/?utm_source=ack\&utm_medium=journal) for editing a draft of this manuscript.

\section{Conflicts of Interest}

The author declares no conflicts of interest regarding the publication of this paper.

\section{References}

[1] Geiger, J.A. (2018) Measurement Quantization Unites Classical and Quantum Physics. Journal of High Energy Physics Gravitation and Cosmology, 4, 262-311. https://doi.org/10.4236/jhepgc.2018.42019

[2] Einstein, A. (1914) Vol. 6: The Berlin Years: Writings, 1914-1917. 30. (English Translation Supplement) http://einsteinpapers.press.princeton.edu/vol6-trans/42

[3] Nicolai, H., Peeters, K. and Zamaklar, M. (2005) Loop Quantum Gravity: An Outside View. Classical and Quantum Gravity, 22, R193.

https://doi.org/10.1088/0264-9381/22/19/R01

[4] SNO Collaboration (2001) Measurement of the Rate of nu_e + d -- > p + p + $\mathrm{e}^{\wedge}$-Interactions Produced by $8 \mathrm{~B}$ Solar Neutrinos at the Sudbury Neutrino Observatory. https://arxiv.org/abs/nucl-ex/0106015

[5] Ellis, G., Van Elst, H. and Lachièze-Rey, M. (Eds.) (1999) Theoretical and Observational Cosmology: Cosmological Models (Cargèse Lectures 1998). In: Theoretical and Observational Cosmology, Proceedings of the NATO Advanced Study Institute on Theoretical and Observational Cosmology, Kluwer, 17-29 August 1998, 1-116. arXiv:gr-qc/9812046

[6] Ashtekar, B. and Lewandowski, J. (2004) Background Independent Quantum Gravity: A Status Report. Classical and Quantum Gravity, 21, 15. https://doi.org/10.1088/0264-9381/21/15/R01

[7] Nicolai, H., Peeters, K. and Zamaklar, M. (2005) Loop Quantum Gravity: An Outside View. Classical and Quantum Gravity, 22, R193-R247. arXiv:hep-th/0501114 https://doi.org/10.1088/0264-9381/22/19/R01

[8] Shwartz, S. and Harris, S.E. (2011) Polarization Entangled Photons at X-Ray Energies. Physical Review Letters, 106, 080501. arXiv: 1012.3499 https://doi.org/10.1103/PhysRevLett.106.080501

[9] Ballentine, L.E. (1970) The Statistical Interpretation of Quantum Mechanics. Reviews of Modern Physics, 42, 358-381. https://doi.org/10.1103/RevModPhys.42.358

[10] Mohr, P., Taylor, B. and Newell, D. (2012) CODATA Recommended Values of the Fundamental Physical Constants: 2010. 73. arXiv:1203.5425v1

[11] Mohr, P., Taylor, B. and Newell, D. (2015) CODATA Recommended Values of the Fundamental Physical Constants: 2014. 3. arXiv: 1507.07956v1

[12] Einstein, A. (1905) Zur Elektrodynamik bewegter Körper, (Electrodynamics of Moving Bodies). Annalen der Physik, 322, 891-921. https://doi.org/10.1002/andp.19053221004

[13] Schutz, B.F. (2003) Gravity from the Ground up. Cambridge University Press, Cambridge, 361. https://doi.org/10.1017/CBO9780511807800

[14] Planck, M. (1899) Über irreversible Strahlungsvorgänge, Sitzungsberichte der 
Königlich Preußischen Akademie der Wissenschaften zu, Berlin, (About Irreversible Radiation Events, Meeting Reports of the Royal Prussian Academy of Sciences). Vol. 5, 480.

[15] Peebles, P.J.E. (1968) Recombination of the Primeval Plasma. Astrophysical Journal, 153, 1. https://doi.org/10.1086/149628

[16] Pais, A. (1979) Einstein and the Quantum Theory. Reviews of Modern Physics, 51, 863-914. https://doi.org/10.1103/RevModPhys.51.863 